\title{
The White Matter Structural Network Underlying Human Tool Use and Tool Understanding
}

\author{
Yanchao Bi, ${ }^{1}$ Zaizhu Han, ${ }^{1}$ Suyu Zhong, ${ }^{1}$ Yujun Ma, ${ }^{2}$ Gaolang Gong, ${ }^{1}$ Ruiwang Huang, ${ }^{3}$ Luping Song, ${ }^{4}$ Yuxing Fang, ${ }^{1}$ \\ Yong $\mathrm{He},{ }^{1}$ and Alfonso Caramazza ${ }^{5,6}$ \\ ${ }^{1}$ State Key Laboratory of Cognitive Neuroscience and Learning and IDG/McGovern Institute for Brain Research, Beijing Normal University, Beijing 100875, \\ China, ${ }^{2}$ Department of Psychology, Henan University, Kaifeng 475000, China, ${ }^{3}$ Center for the Study of Applied Psychology, Key Laboratory of Mental Health \\ and Cognitive Science of Guangdong Province, School of Psychology, South China Normal University, Guangzhou 510631, China, ${ }^{4}$ Neurorehabilitation \\ Department of Capital Medical University Rehabilitation College and China Rehabilitation Research Center, Beijing 100038, China, ${ }^{5}$ Department of \\ Psychology, Harvard University, Cambridge, Massachusetts 02138, and ${ }^{6}$ Center of Mind/Brain Sciences (CIMeC), University of Trento, I-38122 Trento, Italy
}

The ability to recognize, create, and use complex tools is a milestone in human evolution. Widely distributed brain regions in parietal, frontal, and temporal cortices have been implicated in using and understanding tools, but the roles of their anatomical connections in supporting tool use and tool conceptual behaviors are unclear. Using deterministic fiber tracking in healthy participants, we first examined how 14 cortical regions that are consistently activated by tool processing are connected by white matter (WM) tracts. The relationship between the integrity of each of the 33 obtained tracts and tool processing deficits across 86 brain-damaged patients was investigated. WM tract integrity was measured with both lesion percentage (structural imaging) and mean fractional anisotropy (FA) values (diffusion imaging). Behavioral abilities were assessed by a tool use task, a range of conceptual tasks, and control tasks. We found that three left hemisphere tracts connecting frontoparietal and intrafrontal areas overlapping with left superior longitudinal fasciculus are crucial for tool use such that larger lesion and lower mean FA values on these tracts were associated with more severe tool use deficits. These tracts and five additional left hemisphere tracts connecting frontal and temporal/parietal regions, mainly overlapping with left superior longitudinal fasciculus, inferior frontooccipital fasciculus, uncinate fasciculus, and anterior thalamic radiation, are crucial for tool concept processing. Largely consistent results were also obtained using voxel-based symptom mapping analyses. Our results revealed the WM structural networks that support the use and conceptual understanding of tools, providing evidence for the anatomical skeleton of the tool knowledge network.

Key words: brain-damaged patient; diffusion tensor imaging; structural network; tool concept; tool use; voxel-based lesion-symptom mapping

\section{Introduction}

The ability to use complex tools is a milestone in human evolution (Gibson et al., 1994). Understanding the neural circuits supporting the use and knowledge of tools is an important

\footnotetext{
Received Sept. 5, 2014; revised March 5, 2015; accepted March 21, 2015.

Author contributions: Y.B., Z.H., R.H., and A.C. designed research; Y.M. and L.S. performed research; Z.H., S.Z., Y.M., G.G., and Y.F. analyzed data; Y.B., Z.H., Y.H., and A.C. wrote the paper.

This work was supported by the 973 Program (Grants 2013CB837300 and 2014CB846100), Major Project of National Social Science Foundation (Grant 11\&ZD186), the National Natural Science Foundation of China (Grants $31171073,31222024,31221003,81071149,81271548,81371535,31271115$, and 81322021), Program for New Century Excellent Talents in University (Grant 12-0055; 12-0065), Beijing Nova Program (Grant Z121110002512032), Fundamental Research Funds for the (entral Universities (Grant 2014kJJCA07), and Beijing New Medical Discipline Based Group (Grant 100270569). A.C. was supported by the Fondazione Cassa di Risparmio di Trento e Rovereto. We thank Litao Zhu for help with imaging methodology; Bin Gu for neuroimaging data preprocessing; Xiaochun Han, Yingxian Zheng, Xingyu Liu, Yaxing Duan, Yuan Chang, and Huanglin Jiang for scoring tool use data, all BNU-CNLab members for data collection and preprocessing, in particular Yangwen Xu; and all research participants.

The authors declare no competing financial interests.

Correspondence should be addressed to Zaizhu Han, State Key Laboratory of Cognitive Neuroscience and Learning and IDG/McGovern Institute for Brain Research, Beijing Normal University, Beijing 100875, China. E-mail: zzhhan@bnu.edu.cn.

DOI:10.1523/JNEUROSCI.3709-14.2015

Copyright $\odot 2015$ the authors $\quad 0270-6474 / 15 / 356822-14 \$ 15.00 / 0$
}

component of the study of the modern human brain. Much neuropsychological and neuroimaging evidence has identified several brain regions in parietal, frontal, and temporal cortices that are associated with tool processing (De Renzi et al., 1968; Goldenberg and Hagmann, 1998; Rumiati et al., 2001; Johnson-Frey, 2004; Lewis, 2006; Goldenberg and Spatt, 2009; Randerath et al., 2010; Buxbaum et al., 2014; Goldenberg, 2014; Hoeren et al., 2014; Orban and Caruana, 2014). Neuropsychological studies have found that lesions to the left temporal lobe can lead to tool understanding impairment and lesions to the parietal lobe lead to tool use impairment (Johnson-Frey, 2004; Goldenberg and Spatt, 2009; Buxbaum et al., 2014). A meta-analysis (Lewis, 2006) of studies using functional neuroimaging to investigate the activation patterns associated with tool-processing tasks showed that the tasks involving tools understanding primarily activated ventral regions, including fusiform gyrus (FFG), posterior middle temporal gyrus (pMTG), and inferior frontal gyrus (IFG); tasks involving using tools activated dorsal regions including dorsolateral premotor cortex (DLPMC), ventral premotor cortex (VPMC), IFG, inferior parietal lobule (IPL), and superior parietal lobule (SPL), as well as pMTG. The set of regions consistently 


\section{A Mapping behavior with self-obtained tracts}

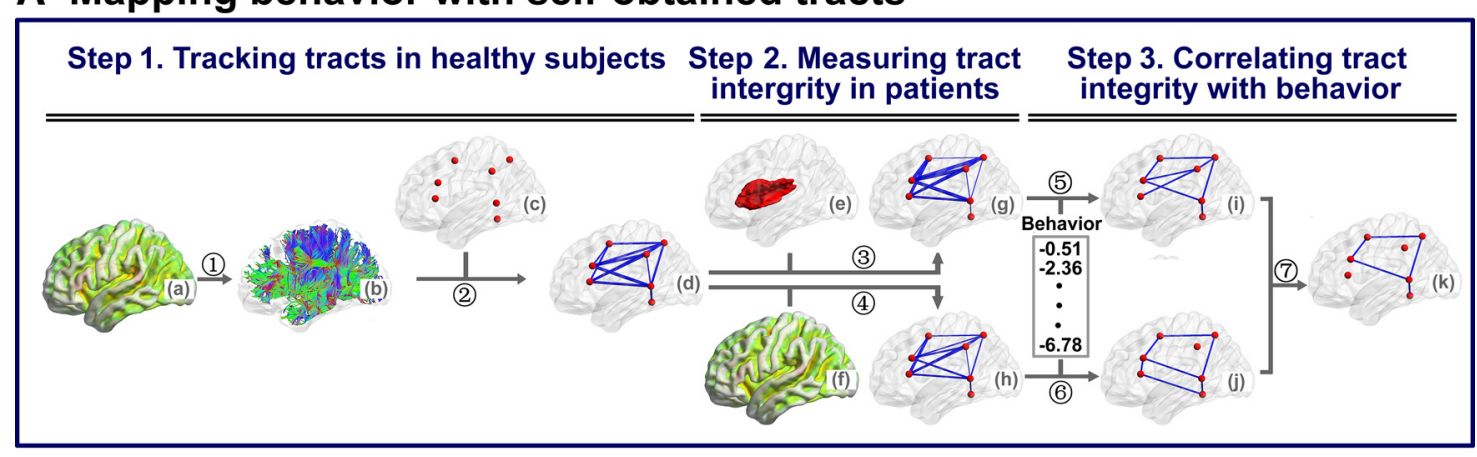

\section{B Validation with voxel-based lesion-symptom mapping analysis}

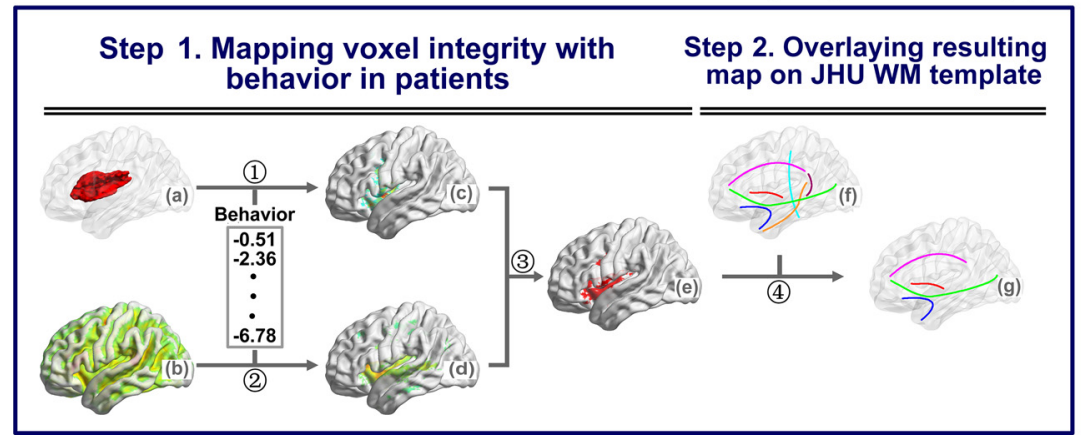

Figure 1. Flowchart for the analyses of behavioral and imaging data. A, Mapping behavior with self-obtained tracts: (1) Reconstruction of white fibers in the whole brain (b) by using DTI deterministic tractography on the FA (fractional anisotropy) map (a) for healthy participants. (2) Filtering out the WM fibers connecting every pair of seed spheres (c) and identifying the tract mask of each pair seeds $(\boldsymbol{d})$ consisted of all the voxels reaching the threshold with AlphaSim correction (corrected cluster level: $p<0.05$; voxel level: sign test $p<0.05$ ). (3-4) Inputting the tract mask into the lesion map $(\boldsymbol{e})$ or the FA map $(\boldsymbol{f})$ of each patient to calculate the percentage of voxels with lesion ( $\boldsymbol{g}$, number of voxels with lesion divided by total number of voxels on the tract) or mean $F A$ value ( $\boldsymbol{h}$, averaging the FA values of all voxels in the tract), respectively. (5-6) Obtaining the tool-relevant tracts in separate analyses, through correlating the standardized " $t$ " behavioral scores with lesion percentages (i) or the mean FA values ( $(j)$ across the 86 patients, partialling out the total lesion volume. (7) Obtaining the tracts important for tool processing, namely, those with significant effects in both the lesion analysis and the mean FA analysis. $\boldsymbol{B}$, Validation with voxel-based lesion-symptom mapping analyses: (1) Calculating VLSM analysis map (c) by comparing the standardized " $t$ " behavioral scores between damaged and intact patients on each voxel of the lesion map (a), with total lesion volume as a covariate. (2) Calculating VFSM analysis map (d) by correlating the standardized " $t$ " behavioral scores with FA values of each voxel of the FA map (b) across the 86 patients, with total lesion volume as a covariate. (3) Obtaining the tool-related voxels (e), which had significant effects in both the VLSM and VFSM analyses. (4) Extracting the tool-related tracts ( $\boldsymbol{g}$ ), through overlaying the tool-related voxels onto JHU WM tractography atlas (Hua et al., 2008), which includes 20 major WM tracts of the human brain. The figures were displayed using the BrainNet Viewer (Xia et al., 2013) software package.

activated in tool processing tasks is often referred to as the tool network, including a dorsal tool use network and a ventral tool concept network.

Although there is a large body of results concerning the cortical regions involved in one way or another in tool processing, much less is known about the structural connection patterns, with no studies directly examining the putative role of such connections in creating a functionally integrated tool-processing network. Seminal evidence for the structural connectivity of the tool network was obtained by Ramayya et al. (2010) and by Hoeren et al. (2013) using diffusion tensor imaging (DTI). Both studies tracked the pathways between predefined regions of interest (ROIs) implicated in tool processing and obtained distinct connections between the ROIs.

Although these studies have provided important insights regarding the structural network of tool processing, the functional role of the tracts putatively involved in tool processing was only inferred on the basis of the presumed functions of the regions they connected. However, the fact that there are white matter (WM) connections between these regions does not necessarily mean that they participate in tool processing: the connected gray matter (GM) regions may participate in different processes associated with tools, but the connections between them may not be directly relevant to those processes. In addition, the activation of one or both GM regions connected by a WM tract might not be functionally necessary in tool processing. Therefore, direct examination of the causal role of WM pathways in tool processing behavior is essential for definitive conclusions about the structural connectivity of the tool knowledge network.

The present study aimed to reconstruct the structural network crucial for tool processing using diffusion and structural imaging data in healthy and brain-damaged patient populations.

\section{Materials and Methods}

Methodology overview

A flowchart of the experiments and analyses is shown in Figure 1. The main idea is to map tool use and tool conceptual performance with WM integrity. Given that our primary interest was to understand how specific tool-relevant GM regions are structurally connected to support tool processing, we examined tracts connecting GM ROIs by tractography using our own WM data with healthy individuals (Fig. 1A). We then validated our findings through voxel-based symptom-mapping analyses, relating the results to the WM tracts in a common template/Johns Hopkins University (JHU) WM tractography atlas (Hua et al., 2008; Schwartz et al., 2012; Fig. 1B). The first approach more directly elucidates the effects of WM connections between specific GM ROIs, which do not necessarily match fully with the template pathways (e.g., being a subsection of a large pathway); the second approach provides a better understanding of the results in the context of classically mapped WM pathways. More specif- 
ically, in the main analysis, we first charted the WM fiber bundles between tool-activated regions across the whole brain by performing deterministic fiber tracking with DTI data in healthy individuals. To determine whether these potential WM tracts contribute to tool processing, we performed a patient study with 86 individuals with brain damage and examined the relationship between the integrity of each of the identified WM tracts and behavioral performance on tool processing. WM tract integrity was measured with both lesion percentage (structural imaging) and mean fractional anisotropy value (diffusion imaging). Both measures have their limitations and advantages: lesion measures do not capture the integrity variations in the voxels without lesion; fractional anisotropy (FA) values may be misleading in voxels with crossing fibers. To be conservative, we considered the conjunction results of these two measures. Behavioral performance was assessed by a tool use task and multiple tasks involving tool concepts (oral picture naming, pictureassociative matching, and word-associative matching). Significant correlation between tract integrity and impairment in tool use or tool concept comprehension, controlling for confounding variables, would indicate a causal effect of the target tract in tool processing. In the voxel-based lesion-symptom mapping validation analysis, tool use or tool concept performance of the 86 patients were first mapped with the integrity/ damage value of each voxel on the whole brain using voxel-based lesionsymptom (VLSM) mapping (structural imaging; Bates et al., 2003) and voxel-based FA-symptom mapping (VFSM; diffusion imaging, see below for details) approaches. The voxels that were statistically significant in VLSM and VFSM analyses were then overlaid onto the JHU WM tractography atlas. Finally, we assessed the degree to which the observed tracts are at least partly specific for tools by testing control tasks (intransitive actions as a control for tool use; animals and people as a control for tool concepts).

\section{Participants}

Behavioral and imaging data were collected for a group of healthy subjects and a group of brain-damaged participants, a similar cohort to that of another study by our group (Han et al., 2013a). All healthy participants $(n=49)$ and 68 of the 86 patients of the current study were included in that previous study. The differences in subject inclusion were because some participants completed behavioral tasks for only one of the two studies. Identical testing procedures were used for the two groups of participants. Participants were all native Chinese speakers and had provided written informed consent. The study was approved by the Institutional Review Board of the State Key Laboratory of Cognitive Neuroscience and Learning, Beijing Normal University.

Healthy participants. Forty-nine healthy adults (26 males) in Beijing participated in the study. The mean age of the group was 50 years $(\mathrm{SD}=$ 11 , range: $26-72)$ and the mean formal education was 13 years $(\mathrm{SD}=4$, range: 6-22). All subjects were right handed (Oldfield, 1971), had normal or corrected-to-normal vision and hearing, and no history of psychiatric or neurological disease. The Chinese version of the Mini-Mental State Examination (MMSE) (Folstein et al., 1975) was used to evaluate the general cognitive state $($ mean $=28, S D=1$, range: $24-30)$. They were paid for their participation.

Patients. Eighty-six brain-damaged patients ( 65 males) were recruited for this study from the China Rehabilitation Research Center. We adopted the following inclusion criteria: had no previous brain injury; had no other neurological or psychiatric disease such as alcohol abuse or severe depression; was at least 1 month post-onset (mean $=6$ months, $\mathrm{SD}=11$, range: $1-86$ ); and had enough language comprehension ability to understand task instructions. All were right handed. The majority suffered from stroke $(n=74)$ and the others from traumatic brain injury $(n=12)$. Thirty-five patients had bilateral lesions, 33 had left hemisphere lesions, and 18 had right hemisphere lesions. The mean age was 45 years $(\mathrm{SD}=13$, range: $19-76)$ and the mean formal education was 13 years $(\mathrm{SD}=3$, range: $2-19)$. The mean score on MMSE was 21 ( $\mathrm{SD}=8$, range: $0-30)$. A comprehensive language screening adopting aphasia classification criteria in Gao (1993) revealed 10 patients without signs of aphasia, 15 with motor aphasia, 8 with sensory aphasia, 12 with anomia, 5 with conduction aphasia, 29 with global/mixed aphasia, and 5 with subcortical aphasia, 2 with dyslexia or dysgraphia.

\section{Behavioral data collection}

Each participant was individually tested in multiple sessions in a quiet room. Each session lasted $<2 \mathrm{~h}$ with pauses for rest upon request.

Tool use task. This task included 10 common tools (scissors, brush, key, chopsticks, saw, broom, fan, iron, match, and glasses). Participants were given each tool in their nonparetic hand and were instructed to perform how it is typically used (e.g., cutting). Their performance was recorded with a digital video recorder and was later viewed and scored. Given the common difficulty in categorizing an action response as correct or error due to variation in various aspects of the action, instead of the conventional binary coding, we first asked two naive independent raters to rate on a 7-point scale how appropriate each tool use action was overall, with the instructions to give 1 to "completely incorrect," 7 to "completely correct," and 4 to "somewhat correct." They were first trained on eight subjects, for which discrepant items were discussed together to reach common criteria. They then independently scored all responses, with high interrater agreement across ratings for all responses of patients $(r=$ $0.90, p<10^{-30}$ ), and the average of the two raters' scores was taken as the performance score for each item. To better understand the nature of the errors, we also performed response analyses closely following Buxbaum and Saffran (2002) and Buxbaum et al. (2005, 2014), scoring each responses as correct or error ( 1 or 0 ) along five dimensions: ( 1 ) content (the target gesture was substituted by another recognizable gesture); (2) hand posture (hand posture/grasp was unrecognizable, flagrantly incorrect, or only transiently correct); (3) arm posture/trajectory (arm posture and/or trajectory, shape of movement, were flagrantly incorrect or only transiently correct); (4) amplitude (size of movement was clearly too large, too small, or transiently correct); and (5) timing/frequency (speed of movement was flagrantly too fast or slow and the number of cycles of movement was flagrantly too few or many). The average of the hand posture and arm/trajectory scores reflects postural accuracy and the average of the amplitude and timing/frequency scores reflect kinematic accuracy. Four new coders were trained on the responses of four patients. After reaching high interrater reliability in percentage agreement (mean: 96\%, range: $93-100 \%$ ) and Cohen's $\kappa$ value (mean: 0.91 ; range: $0.82-1$ ), they each scored about one-fourth of the remaining patients and healthy subjects.

Non-tool-use action imitation task. To test whether patients' failures in the tool use task may arise from impairment in producing actions in general, participants performed an imitation task on non-tool actions. Ten intransitive hand-related actions (e.g., salute, applaud) were performed by an actor and videotaped. Participants were instructed to imitate each action after watching the video. Recording and scoring procedures were identical to those for the tool use task.

Tool concept tasks. Tool conceptual processing ability was assessed by jointly considering performance on three tasks: oral picture naming, picture-associative matching, and word-associative matching. These tasks involve the conceptual component of the tool network but vary in the modalities of input (verbal and nonverbal stimuli) and output (naming and button press) (see similar approach in Jefferies et al., 2008; Schwartz et al., 2012; Wei et al., 2012; Han et al., 2013a). We used the composite score across these three tasks as the tool conceptual score. The rationale is that the composite of these three tasks would likely reflect the common cognitive component (i.e., semantics) rather than taskspecific components (e.g., oral production, picture perception/recognition, word recognition, or button press). A potential problem with this approach is that the composite score may be primarily driven by one or two tasks and as a result reflect some nonsemantic, task-specific input or output process. To cope with this possibility, we adopted another approach for convergence using principal component analysis (PCA) (Jolliffe, 2002) on two sets of tasks: one set included the three conceptual tasks and the other set included the latter tasks as well as two additional tool tasks (oral sound naming, six tool items; oral word reading, 20 tool names) so as to maximize the variation in degree of reliance on various processing components. The weights of the conceptual component extracted from different tasks were examined. There were 20 trials in the oral picture-naming task and 10 in each of the associative matching tasks. In the naming task, participants were visually presented a colored photograph of a common tool (e.g., hammer) on a computer screen and were 
instructed to speak out its name. The first complete response was scored. The two matching tasks had the same format as the Pyramids and Palm Trees Test (Howard and Patterson, 1992). For the picture version, each trial contained the photographs of three tools on a touch screen arranged in an upright triangle. Participants judged which of the two bottom images (e.g., spoon, ruler) was semantically closer to the top one (e.g., chopstick) by touching the corresponding photograph on the screen. The word version was the same as the picture one, except that the pictures were replaced with their written names. There was a $60 \mathrm{~s}$ response deadline for each item in all three tasks. Responses were scored 1 if correct and 0 if wrong or if no response was given within the time deadline. The picture task was tested before the word version; many other unrelated cognitive tasks intervened between them.

Non-tool conceptual tasks. To evaluate the extent of specificity of tract damage on tool conceptual processing performance, we performed parallel conceptual tasks (oral picture naming, picture-associative matching, and word-associative matching) with two other object categories, animals and persons. The same numbers of animal and people items as those used in the tool concept tasks were used. The animal category included common mammals, birds, insects, fish, reptiles, and amphibians. The person category included photographs or names of famous people that are familiar to the Chinese population.

\section{Behavioral data preprocessing for patients}

Behavioral data correction. Because the patient group showed considerable variation in demographic properties (e.g., age, sex, education level), "raw" rating scores (on tool use) or accuracy scores (on conceptual tasks) might not meaningfully reflect the degree of deficit. We adopted a standardization method proposed by Crawford and Garthwaite (2006) in which patients' behavioral scores are corrected by considering the performance distribution in the healthy population and transforming each patient's raw scores into standardized " $t$ " scores. This method has been described in detail in our recent studies (Han et al., 2013a; Han et al., 2013b). In brief, for each task, we first established a regression model on the basis of the properties of the healthy control group, with the dependent variable being their rating or accuracy scores and the predictors (age, sex, and years of formal education). A predicted accuracy score for each patient was acquired by introducing his or her demographic information into the model, and it was used to generate a discrepancy value $\left(\right.$ Discrepancy $\left.y_{\text {patient }}\right)$ (i.e., observed accuracy - predicted accuracy). A corrected SE of estimate for each patient $\left(\mathrm{SE}_{\text {patient }}\right)$ was obtained. The patient's $t$-score was then computed: $t$-score patient $=$ Discrepancy $_{\text {patient }} /$ $\left.\mathrm{SE}_{\text {patient }}\right)$. The $t$-scores for the three conceptual tasks were composited into one conceptual $t$-score by first transforming the $t$-scores to $z$-scores and then averaging the $z$-scores on the three conceptual tasks for each patient.

\section{Imaging data collection}

Participants were scanned at the China Rehabilitation Research Center with a 1.5 T GE Signa Excite MRI scanner. Three types of images were obtained: high-resolution 3D T1-weighted images, T2-weighted fluid attenuation inversion recovery (FLAIR) images, and diffusion-weighted images. The 3D images were acquired with T1-weighted 3D MP-RAGE sequence along sagittal orientation with the following parameters: matrix size $=512 \times 512$, voxel size $=0.49 \times 0.49 \times 0.70 \mathrm{~mm}^{3}$, repetition time $=12.26 \mathrm{~ms}$, echo time $=4.2 \mathrm{~ms}$, inversion time $=400 \mathrm{~ms}$, field of view $=250 \times 250 \mathrm{~mm}^{2}$, flip angle $=15^{\circ}$, slice number $=248$ slices. The T2-weighted FLAIR images were obtained on the axial plane with parameters: matrix size $=512 \times 512$, voxel size $=0.49 \times 0.49 \times 5 \mathrm{~mm}^{3}$, repetition time $=8002 \mathrm{~ms}$, echo time $=127.57 \mathrm{~ms}$, inversion $=2 \mathrm{~s}$, field of view $=250 \times 250 \mathrm{~mm}^{2}$, flip angle $=90^{\circ}$, slice number $=28$ slices. Diffusion-weighted imaging had two separate scans with different diffusion weighting direction sets so that, in total, 32 directions were covered. The parameters for the first acquisition were as follows: 15 diffusion weighting directions, matrix size $=128 \times 128$, voxel size $=1.95 \times$ $1.95 \times 2.6 \mathrm{~mm}^{3}$, repetition time $=13000 \mathrm{~ms}$, echo time $=69.3 \mathrm{~ms}$, inversion time $=0 \mathrm{~s}$, field of view $=250 \times 250 \mathrm{~mm}^{2}$, flip angle $=90^{\circ}$, slice number $=53$ slices. The other acquisition had the same parameters except that it included 17 different directions. The first two volumes were b0 volumes and the b-value of other volumes was $1000 \mathrm{~s} / \mathrm{mm}^{2}$ in each acquisition. All the images except for T2-weighted FLAIR images were scanned twice to improve the quality of images.

\section{Imaging data preprocessing}

Structural MRI data. We first coregistered 3D structural imaging data with a trilinear interpolation method in SPM5 (http://www.fil.ion.ucl.ac. $\mathrm{uk} / \mathrm{spm} / \mathrm{software} / \mathrm{spm} 5$ ) and then averaged them. The T2 FLAIR images were coregistered and resliced to the native space of the averaged 3D images. Two trained staff members manually drew each patient's lesion contour on the averaged 3D images slice by slice, visually referring to T2 FLAIR images. The drawn lesions were checked by an experienced radiologist. Each patient's structural images were resliced into $1 \times 1 \times 1$ $\mathrm{mm}^{3}$ voxel size and then semiautomatically normalized into Talairach space via the "3D Volume Tools" in BrainVoyager QX version 2.0 (www. brainvoyager.com) by marking anatomical landmarks (the anterior commissure, the posterior commissure, the most anterior point, the most posterior point, the superior point, the inferior point, the most right point, and the most left point of the cerebrum. Specifically, we used the Advanced Normalization Tools software (ANTs, http://www.picsl. upenn.edu/ANTS/) to estimate the affine transformation between the native and Talairach spaces, which was further applied to transform the lesion masks into the Talairach space with the "WarpImageMultiTransform" program. The lesion masks were then transformed into the Montreal Neurological Institute (MNI) space.

Diffusion MRI data. For the diffusion-weighted imaging data of each participant, we first merged each of the 15 directions and 17 directions paired sequences into one single $4 \mathrm{D}$ image. Then we preprocessed the images with the PANDA pipeline (Cui et al., 2013), which incorporates FSL (Smith et al., 2004), Pipeline System for Octave and Matlab (PSOM) (Bellec et al., 2012), Diffusion Toolkit (Wang et al., 2007), and MRIcron (Rorden et al., 2007). The preprocessing procedure included the following: (1) BET, skull removal; (2) Eddycorrect, correction of eddy current distortion; and (3) DTIFIT, build diffusion tensor models and obtain the FA maps. We then registered them with the FMRIB FA template in the MNI space using ANTs with a linear rigid affine transformation plus a nonlinear transformation. In the linear affine transform, one affine transform txt file for each participant was obtained using ANTs, and then the "WarpImageMultiTransform" program was executed to produce the FA map in MNI space. In nonlinear transform, a shell script "buildtemplate" was used to obtain more fine-grained normalized FA map of each participant in MNI space.

\section{Constructing the tool structural network in healthy participants}

In this step, we mapped whether there exist WM tracts between those GM regions that, in functional neuroimaging studies with healthy individuals, have been shown to be consistently activated by tool processing tasks.

Tool-relevant GM region selection. To cover regions relevant for tool processing as comprehensively as possible, we adopted the regions reported in the meta-analysis by Lewis (2006), which systematically reviewed 32 functional MRI (fMRI) and positron emission tomography (PET) studies covering a variety of tool processing tasks. In total, there were 335 foci (Fig. 5 in that paper) distributed in bilateral DLPMC, bilateral FFG, bilateral IFG, bilateral IPL, left inferior temporal cortex (ITC), bilateral pMTG, bilateral SPL, and bilateral VPMC. The left ITC cluster and left pMTG cluster were very close and have often been considered to be a common region involved in tool processing (Beauchamp et al., 2002), so we did not include left ITC as a separate cluster in the present study. Note that, although right IFG was not labeled in Lewis' (2006) study, we included this cluster because it has been reported to be activated by tool stimuli (Johnson-Frey et al., 2005; Fairhall and Caramazza, 2013). In total, 14 bilateral homologous regions were selected as the set of tool-relevant GM regions. To obtain the best estimate of the coordinates of these 14 regions, we averaged the coordinates of all activation peak foci reported in Lewis (2006) in each region (obtained from the online supplementary materials link provided in the study). The resulting mean coordinates were converted to MNI space (Fig. 1, Table 1). Given that, with this procedure, most regions were connected into continuous clusters, we adopted the Automated Anatomical Labeling 
Table 1. MNI coordinates of the selected seeds associated with tool processing

\begin{tabular}{rllrrrr}
\hline & Seed & & & \\
\cline { 2 - 3 } & Full name & Abbreviation & & $Y$ & Z \\
\hline 1 & Left dorsolateral premoter cortex & IDLPMC & -21 & 2 & 52 \\
2 & Left fusiform gyrus & IFFG & -32 & -46 & -14 \\
3 & Left inferior gyrus & IIFG & -44 & 24 & 9 \\
4 & Left inferior parietal lobe & IIPL & -47 & -40 & 40 \\
5 & Left posterior middle temporal lobe & IPMTG & -52 & -45 & 4 \\
6 & Left superior parietal lobe & ISPL & -19 & -60 & 53 \\
7 & Left ventral promoter cortex & IVPMC & -40 & 21 & 27 \\
8 & Right dorsolateral premoter cortex & rDLPMC & 25 & -2 & 55 \\
9 & Right fusiform gyrus & rFFG & 30 & -45 & -14 \\
10 & Right inferior gyrus & rIFG & 50 & 26 & 6 \\
11 & Right inferior parietal lobe & rIPL & 49 & -40 & 37 \\
12 & Right posterior middle temporal lobe & rpMTG & 52 & -48 & 8 \\
13 & Right superior parietal lobe & rSPL & 23 & -54 & 57 \\
14 & Right ventral promoter cortex & rVPMC & 38 & 24 & 23 \\
\hline
\end{tabular}

(AAL) atlas (Tzourio-Mazoyer et al., 2002), which parcelled the GM into 90 anatomical regions, to segment the clusters into the regions corresponding to labels given in Lewis (2006), including FFG, IFG, IPL, and SPL. Because DLPMC, VPMC, and pMTG did not have corresponding regions in AAL, we generated a premotor cortex mask (including precentral gyrus, middle frontal gyrus, and supplementary motor area in AAL labels) and split it equally in half (in $z$-axis) into DLPMC and VPMC regions along the $x$-plane in standard space. Similarly, we split the AAL middle temporal gyrus evenly in $y$-axis into three regions along the $z$-plane, with the posterior section being defined as the pMTG region.

With the coordinates of the 14 GM regions obtained, we generated 14 spheres with $15 \mathrm{~mm}$ radius in MNI space. These 14 masks were then transformed back to the native diffusion space for each subject to serve as nodes for the tractography below. IFG sphere and VPMC sphere in both hemispheres had overlapping voxels and tractography was not conducted between these two pairs. In total, 89 node mask pairs were determined.

WM connections extracted from diffusion MRI tractography in healthy participants. For each healthy subject, the whole brain tracts were reconstructed using the FACT tracking algorithm (Mori et al., 1999). Specifically, the fiber tracking was terminated when the angle between two consecutive orientations was greater than a given threshold $\left(45^{\circ}\right)$ or when the FA value was smaller than a given threshold $(0.20)$. Given that the outcome of tractography is affected by the initial position of the seed points within the voxel (Cheng et al., 2012), 100 seeds were randomly selected within each voxel to avoid biases from initial seed positioning. If fibers seeding within a voxel in one node succeed in fiber tracking to another node, that is, terminating in any voxel in the other node, then the two nodes were considered to be connected by this tracking path, with the passing voxels identified. Therefore, for each node pair, for each subject, we obtained a "tract mask" connecting the two nodes containing all passing voxels on any of the tracking paths between these two nodes. This tract mask is referred to as the tract between the two nodes for this subject.

For each pair of node masks, every tract of each subject was projected to the voxels in the native diffusion space, resulting in a voxel-based binary map. The binary map was further transformed to the MNI space. The tract maps of all subjects for this node pair were overlaid to generate a count map in which the value of each voxel represented the number of subjects who had fibers on it. The count map $(n>0)$ was then thresholded using the AlphaSim correction method, with a corrected $p<0.05$ at cluster level (150-4640 voxels, depending on the size/shape of the tract) and $p<0.05$ at voxel level (sign test). The voxel level $p<0.05$ was obtained using a nonparametric sign test for each voxel in the (subject) count map with the null hypothesis that there is no actual connection in the 49 subjects. The clusters passing the threshold were masked as the tract mask between the two regions and were entered in the subsequent analyses. This analysis was done for all node pairs ( 89 potential tracts) and 33 tracts passed the thresholds.

\section{Testing the effects of observed tracts in tool processing} with patients

To assess whether the observed tracts among the tool-relevant seeds have causal effects on tool processing, we examined the extent to which lesions to these tracts led to tool processing impairment. Specifically, we correlated the integrity of each tract and behavioral performance across patients separately for tool use and for tool comprehension tasks. For tract integrity, we used two types of measures based on structural imaging and diffusion imaging, respectively: with structural imaging, we measured the percentage of damaged voxels (number of voxels with lesion divided by total number of voxels on the tract); with diffusion imaging, we calculated the mean FA value (averaging the FA values of all voxels in the tract). For each tract of interest, in separate analyses, we performed partial correlation analyses between the lesion percentages or mean FA values with tool behavioral scores across patients, with each patient's whole lesion volume as covariate. For each of these analyses we adopted the threshold of $q<0.05$ with false discovery rate (FDR) to correct for multiple comparisons and we considered tracts showing significant effects in both lesion percentage and FA analyses as important tracts.

Controlling for the influence of GM. Given that brain lesions rarely affect only WM while sparing the GM, it is important to understand whether the effects of WM tracts in tool processing observed in the above analyses could be fully accounted for by the effect of relevant GM lesions. We performed partial correlation analyses to control for the contribution of GM effects, that is, calculated partial correlations between tract integrity measures (lesion percentage and mean FA values of each tract) and tool behavioral performance (use and comprehension scores) with lesion percentage on GM regions as covariate. We considered two types of measures for GM effects, in separate analyses: (1) the overall lesion volume in the whole brain GM mask and (2) the lesion volume in each of the tool-relevant GM regions. To generate a mask of the whole brain GM, we included voxels with a probability $>0.40$ in the SPM5 template, excluding the cerebellar regions in AAL template because of signal distortion in the cerebellum. The GM areas sensitive to tool process were masked with the 14 seeds that we used above, with each seed expanded into a 15- mmradius sphere. Voxels within the spheres that landed outside of the whole brain GM mask were excluded.

Controlling for the influence of etiology. In this study, we included patients suffering from one of two kinds of brain injury, stroke and traumatic brain injury. We thus performed two further analyses to confirm that our findings were not modulated by etiology. In one analysis, we calculated partial correlation between tract integrity (lesion percentage and mean FA values of each tract) and tool use and comprehension scores, with etiology type and total lesion volume as covariates. In a second analysis, we performed correlation analyses between tract integrity and behavior in only the stroke patients $(n=74)$, with total lesion volume as covariate.

Mapping tool-relevant tracts with template-based tracts. To understand how the tracts we observed are related to the classical major WM pathways, we overlaid each of the tool use tracts or tool concept tracts obtained above onto the JHU WM tractography atlas (http://fsl.fmrib.ox. ac.uk/fsl/fslwiki/Atlases) in FSL. The $25 \%$ threshold subtemplate was used, which contains 20 major tracts (Hua et al., 2008). The overlapping percentage of the observed tracts mask with each template tract was computed using the following formula: the number of common voxels between the observed tract mask and the template tract divided by the number of total voxels in the template tract (see Table 3).

VLSM and VFSM. Figure $1 B$ displays the procedure of this validation analysis. We correlated the tool performance with the integrity of each voxel (structural imaging or diffusion imaging) to obtain voxels that contributed to tool processing. Specifically, for lesion structural images, a VLSM analysis (Bates et al., 2003; Rorden et al., 2007) was conducted on the data of the 86 patients using the NPM (nonparametric mapping) program in MRIcroN. Voxels in which fewer than five patients had lesions were excluded from the analysis. For each voxel entered in the analysis, the patients were divided into the lesion group and the intact group. The behavioral performance indices (tool use scores and tool conceptual scores) were compared between these two groups while controlling for total lesion volume. A nonparametric Brunner-Munzel (BM) 
Table 2. Behavioral performances on the tool tasks

\begin{tabular}{|c|c|c|c|}
\hline \multirow[b]{2}{*}{ Task } & \multirow{2}{*}{$\frac{\text { Healthy subjects }}{\text { Raw score }}$} & \multicolumn{2}{|l|}{ Patients } \\
\hline & & Raw score & Corrected $t$ score \\
\hline Tool use & $6.58(0.23)$ & $5.71(0.80)$ & $-4.04(3.85)$ \\
\hline \multicolumn{4}{|l|}{ Tool concept } \\
\hline Oral picture naming & $98 \%(3 \%)$ & $74 \%(32 \%)$ & $-8.44(10.83)$ \\
\hline Picture associative matching & $96 \%(6 \%)$ & $87 \%(14 \%)$ & $-1.47(2.13)$ \\
\hline Word associative matching & $96 \%(6 \%)$ & $87 \%(15 \%)$ & $-1.21(2.34)$ \\
\hline
\end{tabular}

Raw score for tool use was the mean 7-point-scale rating value; for tool concepts, it was the mean accuracy on each task. The corrected $t$ score of patients reflected the degree of behavioral deficits by taking into consideration the performance of healthy subjects. Numbers in parentheses are SD.

test (Brunner and Munzel, 2000) was performed. To correct for multiple comparisons, the significance threshold was set at FDR corrected $q<$ 0.05. A whole-brain VLSM $z$-map for tool use or tool concept was then obtained. For DTI diffusion images, a VFSM analysis was carried out. FA values of each voxel in the whole brain were correlated with tool use scores and tool concept scores across patients, with total lesion volume as a covariate (FDR-corrected $q<0.05$ ). A whole brain VFSM $r$-map for tool use or tool concept was obtained. The voxels reaching the threshold in both VLSM and VFSM analyses were considered to be important for tool processing (see Fig. 4). We then overlaid these voxels onto the JHU-WM atlas (Hua et al., 2008). The tracts containing the voxels of tool use or tool concept processing were considered to be necessary for tool use or tool concept, respectively.

Testing the functional specificity of tool-processing tracts

In this part of the project, we tested the degree of functional specificity of those tracts that were found to be important for conceptual and use knowledge of tools. We asked two questions: (1) to what extent are the networks for tool use and tool concept processing dissociable? and (2) to what extent do the observed tool use and tool concept networks contribute to the processing of tools as opposed to general action production or conceptual processing?

Dissociation between tool use and conceptual processing. We performed partial correlation analyses between tract integrity (lesion percentage and mean FA values of each tract) and tool use scores, treating tool concept performance scores as covariate. The results of these analyses reflect tracts that are important for tool use beyond tool comprehension. We also performed partial correlation analyses between tract integrity (lesion percentage and mean FA values of each tract) and tool concept composite scores, treating tool use scores as covariate. The results of these analyses individuate the tracts that are important for tool concepts beyond tool use.

Dissociation between tool use and non-tool-use action imitation. For each tract associated with tool use, we correlated its integrity measures (lesion percentage and mean FA values) with tool use scores across patients, with scores on the intransitive action imitation task as a covariate.

Dissociation between tool conceptual processing and other object categories. For each tract that was found to be critical for tool concept processing, we computed partial correlations between its integrity measures (lesion percentage and mean FA values) and tool concept composite scores, with conceptual composite scores for the animal and person categories as covariates.

\section{Results}

\section{Behavioral performance}

The raw behavioral performance of both healthy and patient groups, as well as the corrected $t$ scores of the patients, is presented in Table 2 . The patients performed significantly worse than healthy subjects in all three tool concept tasks (raw accuracy: oral picture naming: $t=7.18, p<3^{*} 10^{-10}$; picture-associative matching: $t=4.90, p<3 * 10^{-6}$; word-associative matching: $t=$ 4.80, $p<5^{\star} 10^{-6}$ ) and the tool use task (raw rating score: $t=$ 9.43, $p<1 * 10^{-15}$ ). Following Buxbaum et al.'s $(2002,2005$, 2014) scoring procedure, we found that, in the tool use task, the patients made more arm posture/trajectory errors [9\% (the num- ber of error items/that of total items) vs $2 \%, p<0.01$ ], more amplitude errors ( $8 \%$ vs $0 \%, p<0.01$ ), and similar percentages of other types of errors (content errors: $1 \%$ vs $0.4 \%$; hand posture errors: $8 \%$ vs $7 \%$; timing/frequency errors: $3 \%$ vs $2 \% ; p>0.10$ ) relative to healthy subjects. To assess whether the results with this analytic scoring scheme were consistent with those obtained with the 1-7 point rating scheme, we first obtained a composite analytic score by averaging the error scores hand posture/trajectory, arm posture, amplitude, and timing/frequency (following Buxbaum and Saffran, 2002) for each item and correlated these scores with the ones obtained with the 1-7 point rating scheme. The resulting correlation was highly significant $(r=0.67, p<2$ * $10^{-12}$ ), indicating the general reliability across scoring methods. The PCA analysis on the three tool conceptual tasks (oral picture naming, word-associative matching, and picture-associative matching) extracted one component that best captured (55\%) the variance across these tasks, with comparable loadings on the tasks: oral picture naming (0.69), word-associative matching (0.85), and picture-associative matching (0.68), likely reflecting the conceptual component that is commonly shared across these tasks. For each subject, the conceptual PCA score was the sum of his or her performance across the three tasks weighted by the corresponding PCA component loading. The resulting tool conceptual PCA score correlated highly with the composite score described above (the average of the three conceptual tasks) across patients $(r=0.99)$. The PCA analysis including the three conceptual tasks and two additional tool tasks (oral sound naming, oral word reading) extracted two components that best captured (64\%) the variance across the five tasks: one had high loadings on word-associative matching (0.80), oral picture naming (0.72), oral sound naming (0.72), picture-associative matching (0.63), and minimal loading on oral word reading (0.03); the other component had high loading on oral word reading $(0.90)$ and low loading on word-associative matching (0.15), picture-associative matching $(0.05)$, oral sound naming $(-0.46)$, and oral picture naming $(-0.32)$. Therefore, the first component likely reflects conceptual processing and the second probably reflects orthography-phonology-conversion processing. The tool conceptual PCA score (component 1 ) on the 5 tasks again correlated highly with the composite score in the original method $(r=$ 0.97). For simplicity, we only used the composite score of tool conceptual processing in the following analyses.

\section{Tool processing tracts obtained with tractography in healthy participants}

We selected $14 \mathrm{GM}$ seed regions on the basis of a meta-analysis of previous fMRI and PET studies of tool processing (Fig. 2E, Table 1) and tracked the WM fiber bundles between the seed regions in 49 healthy individuals. That is, to estimate whether there existed a WM tract between each seed pair, with theoretically 89 possible connections in total (two connections, IIFG-IVPMC and rIFGrVPMC, were not tested because the seed pairs had overlapping voxels), we obtained 33 effective tracts reaching the threshold of Alphasim correction (cluster level: $p<0.05$; voxel level: sign test $p<0.05$; Fig. 2E, Table 3). The size of the tracts was $7515 \mathrm{~mm}^{3}$ on average (SD: $6219 \mathrm{~mm}^{3}$, see Table 3). The tracts were generally symmetrical across hemispheres. There were 26 intrahemisphere tracts: 13 in the left hemisphere and 13 in the right hemisphere. Twelve were symmetric and appeared in both hemispheres: DLPMC-IFG, DLPMC-SPL, DLPMC-VPMC, FFG-pMTG, IFGIPL, IFG-SPL, IPL-pMTG, IPL-SPL, IPL-VPMC, pMTG-SPL, pMTG-VPMC, and SPL-VPMC. The other two intrahemisphere tracts were IIFG-lpMTG and rDLPMC-rIPL. Four of seven inter- 
A Lesion overlay map of patients

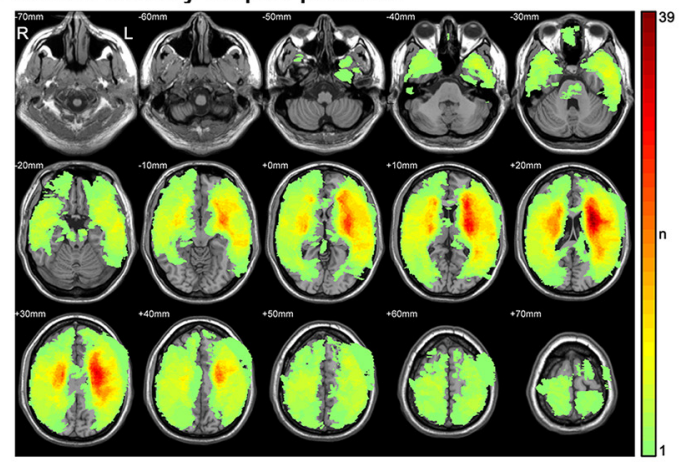

C FA map of healthy subjects

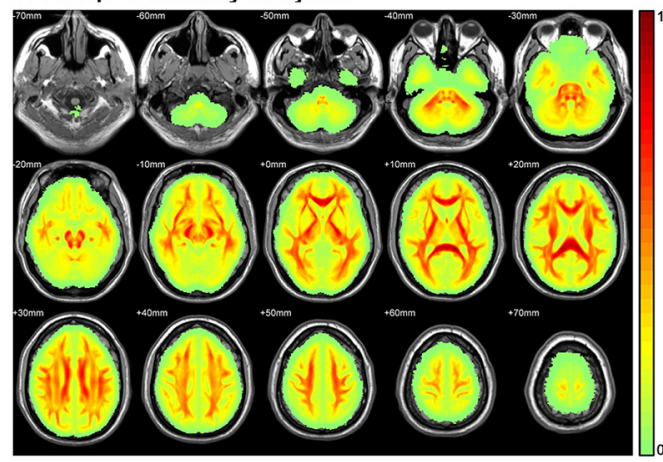

B FA map of patients

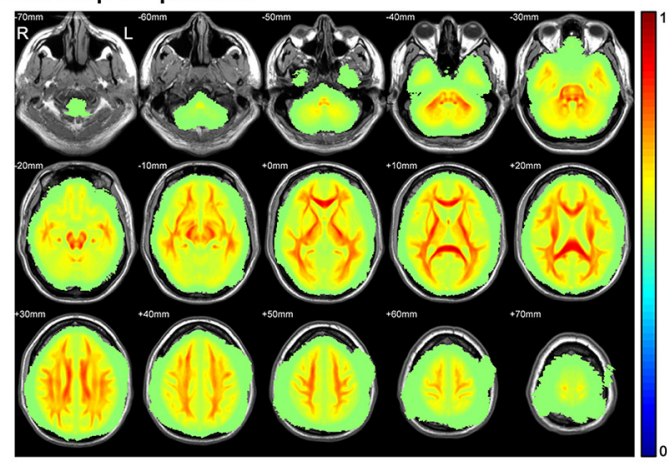

D FA difference map of healthy subjects vs. patients

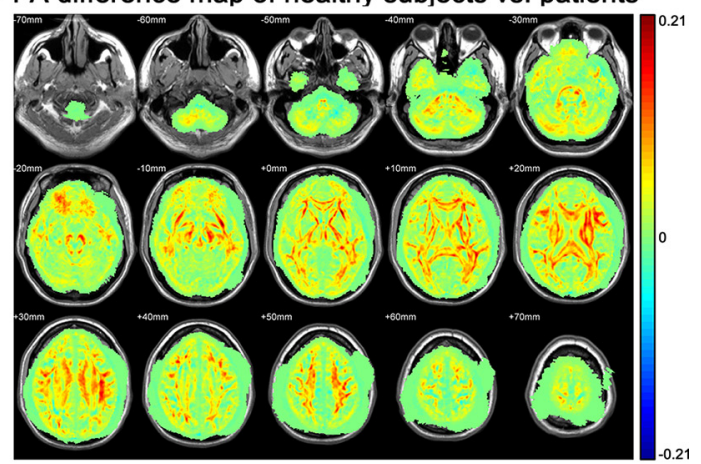

E Effective tracts and mean FA value on them in healthy participants
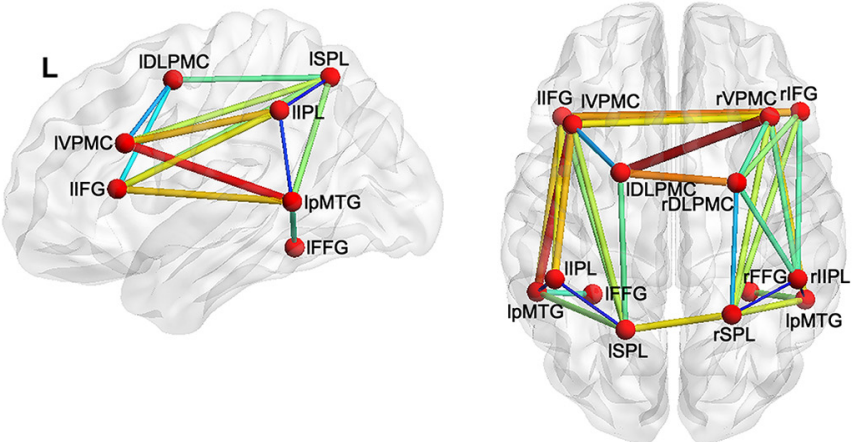

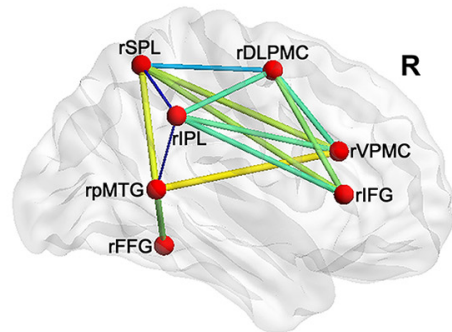

Mean FA value

Figure 2. Raw imaging schematic of participants and the tracts successfully tracked between the tool-relevant seeds. $A$, Lesion map of patients on each voxel. The value of each voxel is the number of patients with lesions on it. $\boldsymbol{B}$, Mean FA maps of patients. $\boldsymbol{C}$, Mean FA maps of healthy subjects. $\boldsymbol{D}$, Mean FA difference map of healthy subjects minus patients. For $\boldsymbol{B}-\boldsymbol{D}$, the color bar indicates the mean FA value per voxel. $\boldsymbol{E}$, Fourteen seeds and 33 tracts successfully tracked between these seeds in 49 healthy adults. The thickness and color of the tracts indicate the mean $F A$ values. Full names of the seeds are listed in Table 1.

hemisphere tracts connected homologous regions: DLPMCs, IFGs, SPLs, and VPMCs. The other three interhemisphere tracts were IDLPMC-rVPMC, IIFG-rVPMC, and IVPMC-rIFG.

Note that, although the ROIs have the same size (15 mm radius spheres), the number of WM voxels in each ROI sphere varied [under the threshold FA value $>0.15$ for the WM mask of our own 49 healthy subjects, WM voxel percentage range: $32-$ 91\%; across ROIs $\left.\chi^{2}(13)=7079, p<0.0001\right]$, which may lead to different chances of tracking success. To determine whether the 33 tracts were obtained simply because their corresponding ROIs contained greater WM, we compared the WM size of the ROI pairs with connecting tracts with the ROI pairs without connecting tracts in our results. Specifically, for the two ROIs of each of the obtained tracts, we computed the mean, minimum, and maximum number of WM voxels of the pair. We also computed the same measures of WM voxels for the ROI pairs for which no tracts were obtained. $t$ tests comparing the number of WM voxels in the two types of ROIs revealed no significant differences for the ROI-means or the ROI-min values of the two groups $(p>0.30)$ and the opposite-to-predicted trend of the ROI-max values (with tracts pairs < no tract pairs in WM size: 10381 vs 11178 voxels). Within the 33 obtained tracts, there was no significant correlation between the WM size of the ROIs and the size of the tracts $\left(R_{\mathrm{ROI} \text {-mean }}=0.18 ; R_{\mathrm{ROI}-\text { min }}=0.18 ; R_{\mathrm{ROI} \text { - } \max }=0.11\right.$; $p>0.30)$.

Effects of observed tracts in tool processing with patients We tested whether the observed tracts between the tool-activated seeds are functionally necessary for tool processing by correlating tract integrity (lesion percentage and mean FA values) and behavioral performance on tool use and tool concept tasks for the 86 brain-damaged subjects. 
Table 3. Properties of the 33 WM tracts successfully tracked on our healthy subjects and their relationships with tool processing, reflected by the partial correlation coefficients between the tract integrity and the tool use or conceptual performances across patients, with total lesion volume as a covariate

\begin{tabular}{|c|c|c|c|c|c|c|c|c|}
\hline & \multirow[b]{2}{*}{ Tract } & \multirow[b]{2}{*}{ Tract size $\left(\mathrm{mm}^{3}\right)$} & \multirow{2}{*}{$\begin{array}{l}\text { No. of patients } \\
\text { with lesion }\end{array}$} & \multicolumn{2}{|c|}{ Tool use (partial R) } & \multicolumn{2}{|c|}{ Tool concept (partial R) } & \multirow[b]{2}{*}{ Overlay percentage on JHU template tract } \\
\hline & & & & Lesion analysis & FA analysis & Lesion analysis & FA analysis & \\
\hline 1 & IDLPMC-IIFG & 9143 & 52 & $-0.47^{* * *}$ & $0.38^{*}$ & $-0.43^{* * *}$ & $0.38^{* *}$ & ISLF: $0.03 \%(A)$ \\
\hline 2 & IDLPMC-ISPL & 4369 & 26 & -0.08 & 0.16 & -0.01 & 0.07 & \\
\hline 3 & IDLPMC-IVPMC & 13612 & 52 & $-0.46^{* *}$ & $0.39^{* *}$ & $-0.40^{* *}$ & $0.35^{* *}$ & ISLF: $0.15 \%(A)$ \\
\hline 4 & IDLPMC-rDLPMC & 16596 & 60 & -0.08 & 0.18 & -0.01 & 0.07 & \\
\hline 5 & IDLPMC-rVPMC & 1396 & 47 & 0 & 0.13 & 0.2 & 0 & \\
\hline 6 & IFFG-IpMTG & 10725 & 31 & 0.15 & 0.07 & -0.06 & $0.39 * *$ & \\
\hline 7 & IIFG-IIPL & 1653 & 55 & $-0.41^{* *}$ & $0.32^{*}$ & $-0.47^{* * *}$ & $0.45^{* * *}$ & ISLF:13\% (A, M) \\
\hline 8 & IIFG-IpMTG & 2107 & 46 & $-0.30^{*}$ & 0.23 & $-0.54^{* * *}$ & $0.47^{* * *}$ & $\begin{array}{l}\text { IUF: } 19 \% \text { (A); IIFOF: } 16 \% \text { (A, M); IILF: } 2 \% \text { (M, P); ISLF: } 2 \% \text { (P); } \\
\quad \text { IATR:0.09\% (P) }\end{array}$ \\
\hline 9 & IIFG-ISPL & 10811 & 53 & $-0.29 *$ & 0.2 & $-0.49^{* * *}$ & $0.42^{* * *}$ & IIF0F: 32\% (A); IUF: 25\% (A); ISLF: 3\% (P); IATR: 1\% (A) \\
\hline 10 & IIFG-rIFG & 4668 & 61 & $-0.37^{* *}$ & 0.17 & -0.15 & 0.09 & \\
\hline 11 & IIFG-rVPMC & 10103 & 64 & $-0.39 * *$ & 0.15 & -0.17 & 0.07 & \\
\hline 12 & IIPL-IpMTG & 17692 & 41 & -0.08 & 0.09 & -0.18 & 0.32 & \\
\hline 13 & IIPL-ISPL & 5919 & 29 & 0.07 & 0.14 & 0.04 & 0.18 & \\
\hline 14 & IIPL-IVPMC & 2977 & 50 & $-0.39^{* *}$ & 0.26 & $-0.41^{* * *}$ & $0.38^{* *}$ & ISLF: 23\% (A, M) \\
\hline 15 & IpMTG-ISPL & 5891 & 36 & -0.08 & 0.05 & -0.23 & 0.23 & \\
\hline 16 & IpMTG-IVPMC & 2345 & 52 & -0.36 & 0.19 & $-0.43^{* * *}$ & $0.40^{* *}$ & ISLF: 17\% (A, M, P); ISLFT: 1\% (M, P) \\
\hline 17 & ISPL-IVPMC & 4658 & 54 & -0.25 & 0.16 & $-0.47^{* * *}$ & $0.39^{* *}$ & $\begin{array}{l}\text { IIFOF: } 10 \%(A, M) ; \text { IUF: } 9 \%(A) ; \text { ISLF: } 1 \%(P) ; \\
\quad \text { IATR: } 0.02 \%(A)\end{array}$ \\
\hline 18 & ISPL-rSPL & 30391 & 50 & 0.07 & 0.07 & 0.05 & 0.05 & \\
\hline 19 & IVPMC-rIFG & 6993 & 61 & $-0.36^{*}$ & 0.17 & -0.13 & 0.09 & \\
\hline 20 & IVPMC-rVPMC & 13451 & 65 & $-0.38^{* *}$ & 0.17 & -0.15 & 0.09 & \\
\hline 21 & rDLPMC-rIFG & 2192 & 27 & 0.21 & 0.01 & $0.29^{* a}$ & -0.13 & \\
\hline 22 & rDLPMC-rIPL & 513 & 16 & 0.18 & 0.11 & 0.16 & -0.07 & \\
\hline 23 & rDLPMC-rSPL & 1877 & 14 & 0.19 & 0.05 & 0.15 & -0.08 & \\
\hline 24 & rDLPMC-rVPMC & 6955 & 32 & 0.24 & 0 & $0.28^{* a}$ & -0.17 & \\
\hline 25 & rFFG-rpMTG & 2337 & 9 & 0.11 & 0.01 & 0.12 & -0.06 & \\
\hline 26 & rIFG-rIPL & 4292 & 25 & 0.23 & 0.04 & $0.29^{* a}$ & -0.21 & \\
\hline 27 & rIFG-rSPL & 7208 & 39 & 0.13 & -0.05 & $0.32^{* a}$ & -0.23 & \\
\hline 28 & rIPL-rpMTG & 16251 & 20 & $0.26^{* a}$ & 0.1 & 0.21 & 0.02 & \\
\hline 29 & rIPL-rSPL & 8302 & 18 & 0.2 & 0.08 & 0.15 & -0.11 & \\
\hline 30 & rIPL-rVPMC & 5759 & 27 & 0.23 & 0.03 & $0.29^{* a}$ & -0.23 & \\
\hline 31 & rpMTG-rSPL & 7706 & 21 & 0.14 & -0.02 & 0.17 & -0.17 & \\
\hline 32 & rpMTG-rVPMC & 3835 & 24 & 0.2 & -0.01 & 0.27 & -0.24 & \\
\hline 33 & rSPL-rVPMC & 5270 & 37 & 0.11 & -0.04 & $0.30^{* a}$ & -0.23 & \\
\hline
\end{tabular}

The value of tract integrity was the lesion volume percentage (lesion analysis) or mean fractional anisotropy value (FA analysis). FDR corrected: ${ }^{*} p<0.05,{ }^{* *} p<0.01,{ }^{* * *} p<0.001$.

${ }^{a}$ The tract showed effect in the direction opposite to expectation, that is, larger lesion or lower FA values associated with better tool use or comprehension performance. The last column shows the overlay percentage of our tracts of interest with the major tract on the JHU white matter tractography template. Only those showing significant correlation with tool behavior are presented. The letters in parentheses in the last column show the rough locations of the observed tracts in the template tracts ( $\mathrm{A}$, anterior; $\mathrm{M}$, middle; $\mathrm{P}$, posterior). Full names of the tract seeds are given in Table 1.

The lesion and mean FA maps derived from structural and diffusion scans are displayed in Figure 2, $A-D$. The lesions were distributed widely, covering most WM and GM areas, with most patients having lesions in insula and its surrounding WM tissues (Fig. 2A). Although the mean FA map of the patients shows the basic WM connectivity skeleton, the FA values were significantly reduced compared with those of the healthy adults (Fig. $2 B-D$ ). Worth noting is that the number of patients with lesion on each tract varied (Table 3), resulting in statistical power differences in our analyses. There were 15 tracts damaged in $>50 \%$ of the patients (IDLPMC-IIFG, IDLPMC-IVPMC, IDIPMC-rDLPMC, IDIPMCrVPMC，IIFG-IIPL，IIFG-IpMTG，IIFG-ISPL，IIFG-rIFG，IIFGrVPMC, IIPL-IVPMC, IpMTG-IVPMC, ISPL-IVPMC, ISPL-rSPL, IVPMC-rIFG, and IVPMC-rVPMC) and 10 tracts in $>30 \%$ of the patients (IDLPMC-ISPL, IFFG-IpMTG, IIPL-IpMTG, IIPL-ISPL, lpMTG-ISPL, rDLPMC-rIFG, rDLPMC-rVPMC, rIFG-rSPL, rIPLrVPMC, rSPL-rVPMC). The other eight tracts were damaged in $<30 \%$ of the patients. Note that the size of the tract (number of voxels) did not correlate significantly with the chance of being lesioned in our patients (number of patients with lesion on the tract) across the 33 obtained tracts $(r=0.28 ; p=0.11)$.

The results of the correlation analyses between tract integrity measures (lesion percentage and mean FA values) and tool be- havioral performance for the 33 tracts are presented in Figure 3 and Table 3. All analyses controlled for total lesion volume.

For tool use, the lesion percentage analysis revealed 11 tracts with lesion percentages that were significantly negatively correlated with tool use scores (partial $r:-0.47$ to -0.29 , FDR $q<0.05$ ): 3 left frontoparietal tracts (IIFG-IIPL, IIFG-ISPL, IIPL-IVPMC), 2 left frontotemporal tract (IIFG-lpMTG, lpMTG-IVPMC), 2 left intrafrontal tracts (IDLPMC-IIFG, IDLPMC-IVPMC), and 4 interhemisphere tracts (IIFG-rIFG, IIFG-rVPMC, IVPMC-rIFG, IVPMC-rVPMC). The FA analysis revealed three tracts having significant effects: IDLPMCIIFG: partial $r=0.38, q<0.05$; IDLPMC-IVPMC: $r=0.39, q<0.01$; IIFG-IIPL: $r=0.32, q<0.05$ ). These three tracts, all showing convergence across lesion and $\mathrm{FA}$ analyses, are considered further below (Fig. $3 A, B$ ).

Our second coding scheme, which scored each tool use response along multiple dimensions, allows for further elucidation of the role of relevant tracts in the subcomponents of tool actions. We correlated the integrity values (lesion percentages, mean FA values) of the relevant tracts with the postural and kinematic scores across patients, covarying total lesion volume. Greater damage of the left frontoparietal tract (IIFG-IIPL) was associated with lower kinematic scores (lesion analysis: partial $r=-0.32$, $p<0.003$ : FA analysis: partial $r=0.32, p<0.004)$ and marginally 


\section{A Tract schematic}

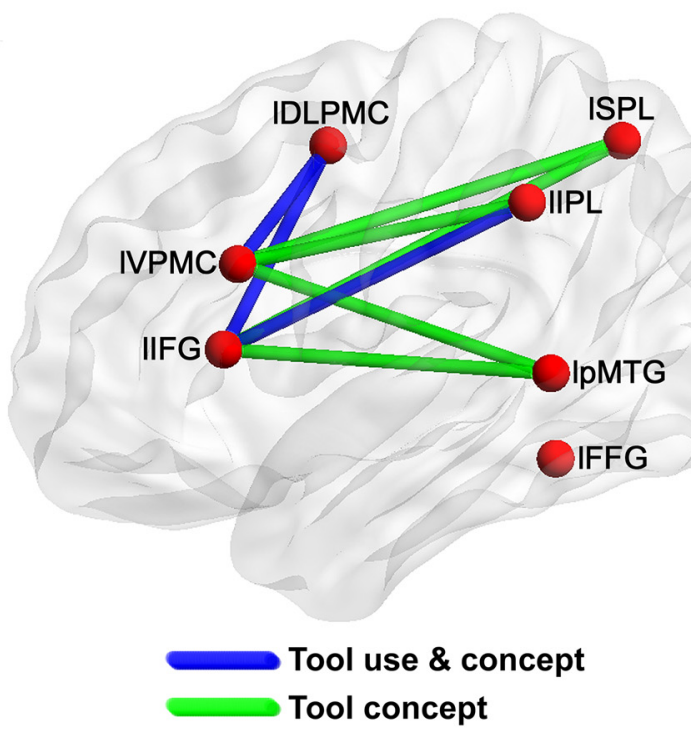

\section{B Tool use}

IDLPMC-IIFG

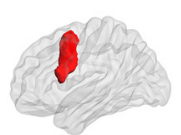

IDLPMC-IVPMC
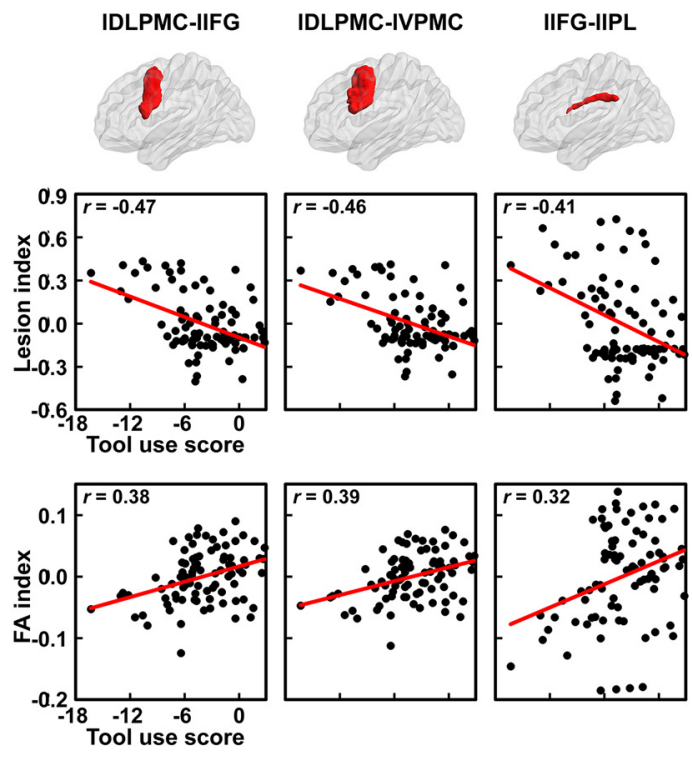

\section{Tool concept}
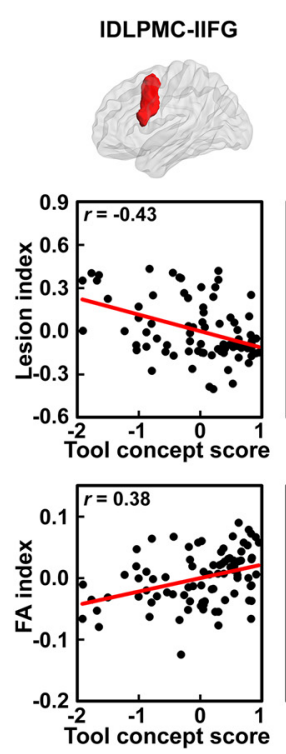
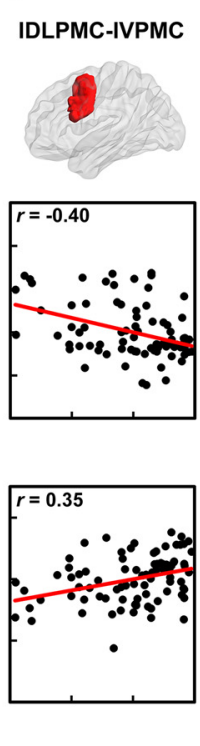
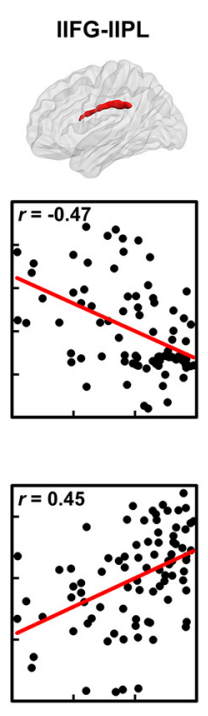
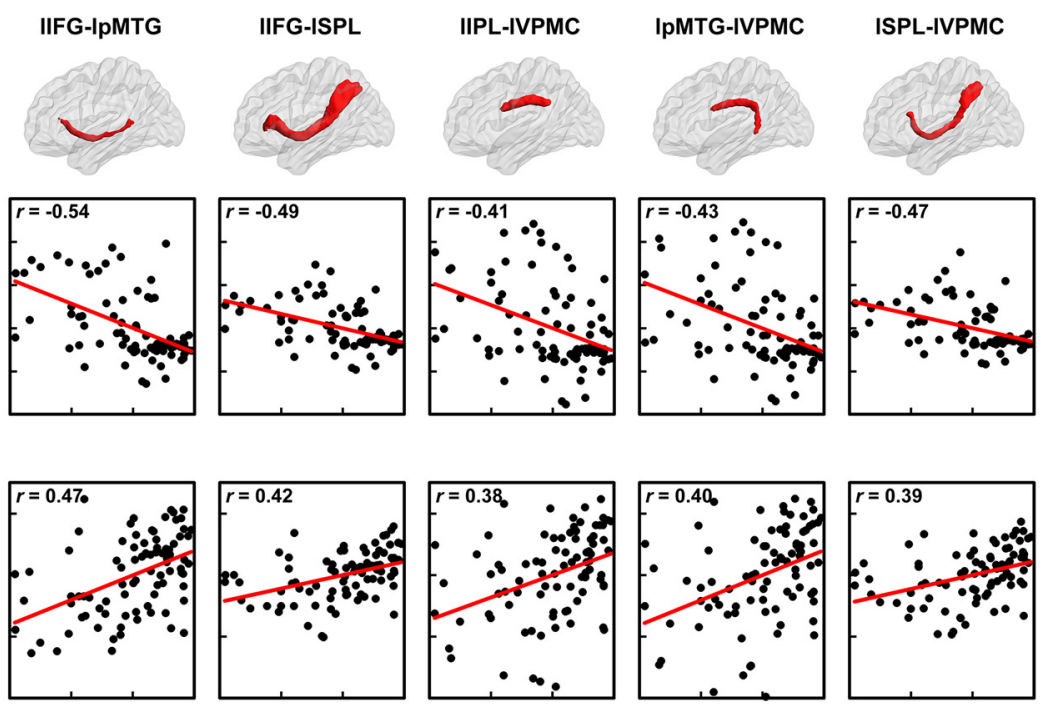

Figure 3. Results of mapping tool-processing behavior with self-obtained WM tracts. $A$, Schematic of the self-obtained WM tracts associated with tool use and/or tool conceptual processing. The correlations of WM tract integrity with tool use or tool conceptual performance are shown in $\boldsymbol{B}$ and $\boldsymbol{C}$, respectively. Row 1 shows the shape of the tracts. Rows 2 and 3 show the correlograms of lesion analysis and FA analysis, respectively. The $y$-axis in the correlograms indicates the residual of the lesion volume (Lesion index) or mean FA value (FA index) with patients' total lesion volume regressed out. Full names of the seeds are listed in Table 1.

less so with postural scores (lesion analysis: partial $r=-0.27, p<$ 0.02: FA analysis: partial $r=0.20, p=0.06)$. The two intrafrontal tracts exhibited significant association with postural scores (IDLPMC-lIFG tracts: lesion analysis: partial $r=-0.31, p<$ 0.005; FA analysis: partial $r=0.29, p<0.008$; IDLPMC-lVPMC: lesion analysis: partial $r=-0.32, p<0.003$; FA analysis: partial $r=0.28, p<0.009$ ) and less so with kinematic scores (IDLPMCIIFG tracts: lesion analysis: partial $r=-0.20, p=0.06$; FA analysis: partial $r=0.17, p=0.11$; IDLPMC-IVPMC: lesion analysis: partial $r=-0.20, p=0.07$ : FA analysis: partial $r=0.17, p<0.11$ ).

For tool concept processing, the integrity of eight tracts was correlated significantly negatively with tool concept composite scores in the lesion analysis: larger lesions on these tracts were associated with worse tool concept performance (partial $r$ : -0.54 to -0.40 , FDR $q<0.01$ ): 4 left frontoparietal tracts (IIFG-IIPL, IIFG-1SPL, IIPL-IVPMC, ISPL-IVPMC), 2 left frontotemporal tracts (IIFG-lpMTG, lpMTG-lVPMC), and 2 left intrafrontal tracts (IDLPMC-IIFG, IDLPMC-IVPMC). The FA analysis revealed 9 tracts with mean FA values that were significantly positively correlated with tool concept scores (partial $r$ : $0.38-0.47$, $q<0.05$ ), including all tracts observed in the lesion analyses except for the lFFG-lpMTG. Below, we focus on the eight tracts obtained in both the lesion and FA analyses (Fig. $3 \mathrm{~A}, \mathrm{C}$ ).

To summarize, three tracts connecting left frontal and parietal and intrafrontal seeds were found to be crucial for tool use; the same three tracts and five additional left frontal-temporal/parietal tracts were crucial for tool conceptual processing. 
Controlling for the influence of GM

To determine whether the effects observed for WM tracts were driven by GM damage, we performed further lesion and FA analyses controlling for the contribution of GM. When lesion percentage on the whole brain GM mask was included as a covariate, the effects of the 3 tracts for tool use obtained in the above analyses were still significant in both the lesion analysis (partial $r$ : -0.50 to $-0.43, p<0.00004$ ) and the FA analysis (partial $r$ : $0.37-0.46, p<0.0006$ ). Similarly, the effects of the 8 tool concept tracts also remained significant in both the lesion analysis (partial $r:-0.54$ to $-0.41, p<0.0002$ ) and the FA analysis (partial $r$ : $0.36-0.46, p<0.0007)$. In the second analysis, we considered the effects of the specific GM regions that were implicated in tool processing, that is, arbitrarily sized $15-\mathrm{mm}$-radius spheres (minus the WM voxels) around the 14 tool-relevant regions that served as seeds in our tracking analyses. When including patients' lesion percentages on each of these 14 seeds as covariates, the correlation between the integrity of the 3 tool use tracts and tool use score remained significant in both the lesion analysis (partial $r:-0.52$ to $-0.49, p<0.00002$ ) and the FA analysis (partial $r$ : $0.38-0.43, p<0.002$ ). The effects of the 8 tool concept tracts also remained significant in both the lesion analysis (partial $r:-0.52$ to $-0.41, p<0.0004$ ) and the FA analysis (partial $r: 0.37-0.47$, $p<0.002)$.

\section{Controlling for the influence of etiology}

We considered the effects of etiology in two kinds of analyses. First, when including etiology type in the partial correlation analyses as a covariate in addition to total lesion volume, the effects of the 3 tool use tracts remained significant in both the lesion analysis (partial $r:-0.43$ to $-0.35, p<0.0009$ ) and the FA analysis (partial $r: 0.28-0.36, p<0.02$ ), as did the 8 tool concept tracts (lesion analysis: partial $r:-0.54$ to $-0.39, p<0.0003$; FA analysis: partial $r$ : $0.37-0.47, p<0.0006)$. Second, we performed the lesion and FA analyses in only the stroke patients controlling for total lesion volume, and all effects held up: tool use tracts lesion analysis: partial $r:-0.46$ to $-0.38, p<0.0009$; FA analysis: partial $r: 0.30-0.36 ; p<0.01$; tool concept tracts lesion analysis: partial $r:-0.56$ to $-0.41, p<0.0003$; FA analysis: partial $r: 0.36-0.48$, $p<0.002$.

\section{Relating the tool-relevant tracts with template-based major WM pathways}

To understand the WM basis of tool processing in the context of major WM pathways that have been commonly established, we tested how our observed tracts are related to template-based major WM pathways. The three tracts that were important for tool use in the above analyses had common voxels with the left superior longitudinal fasciculus (ISLF) of the JHU WM template. The eight tool concept tracts in the above analyses had common voxels with ISLF, left inferior frontooccipital fasciculus (IIFOF), left uncinate fasciculus (IUF), and minimally with left anterior thalamic radiation (IATR), left inferior longitudinal fasciculus (IILF), and left superior longitudinal fasciculus-temporal part (ISLFT) (Table 3).

\section{Validation with VLSM and VFSM approaches}

We further performed VLSM (for structural images) and VFSM (for DTI diffusion images) analyses of tool use and tool concept (FDR corrected $q<0.05$; regressing out total lesion volume) and overlaid the results with the JHU WM template. The results are displayed in Figure 4. The VLSM results on tool use were strongly left lateralized, encompassing the left insula and the left putamen and the underlying WM, indicating that lesions in these territo- ries significantly associated with tool use deficits. The VFSM results on the tool use task rendered the WM between the left putamen and the left frontal lobe. When the common voxels between the above VLSM and VFSM results (mask size: 252 $\mathrm{mm}^{3}$ ) were overlaid on the JHU template, they had overlap with only left SLF ( $0.08 \%$, overlay percentage). For tool concepts, the VLSM results encompassed the left insula, putamen, IFG, and superior temporal gyrus, and their underlying WM; the VFSM results involved the left WM underlying putamen, and right lateral posterior temporal lobe. The common voxels of VLSM and VFSM results (mask size: $8729 \mathrm{~mm}^{3}$ ) overlapped with four left tracts of the JHU template, including IATR (11\%), IIFOF (11\%), ISLF (2\%), and IUF (15\%). Intriguingly, the VFSM results were weaker than the VLSM results overall. One possibility is that FA is only one measure of tract integrity, that is, the degree of anisotropy of diffusion processing, and there are other properties of a tract that are not reflected by FA but can be better captured by lesion status, thus rendering lesion a more sensitive measure for current purposes.

\section{Testing the functional specificity of tool-processing tracts}

Dissociation between tool use and tool concept processing

To reveal tracts that might contribute (partly) unique variables to tool use or tool concepts, we performed partial correlation analyses treating one type of behavioral task as of interest and the other as covariate. When partialling out tool concept composite scores, the effects of the 3 tool use tracts on tool use scores remained significant or marginally significant in both the lesion analysis (partial $r$ : -0.39 to $-0.28, p<0.009$ ) and the FA analysis (partial $r$ : $0.21-0.34, p<0.06$ ). When factoring out tool use scores, the effects of the 8 tool concept tracts remained significant or marginally significant in both the lesion analysis (partial $r$ : -0.47 to $-0.24, p<0.02$ ) and the FA analysis (partial $r: 0.20$ $0.40, p<0.07$ ) (Table 4). These results suggest that the tracts individuated in tool use and tool concept processing did not share all of their underlying substrates/mechanisms.

To further consider whether such potential "dissociations" are due to a difference between motor versus nonmotor tasks, we tested the tool-specific variance of the tool use task (regressed out the non-tool motor task) with the tool concept tasks. We obtained the residual of the tool use task regressing out the non-tool motor control task (i.e., intransitive action imitation). We then examined the correlation between this tool use residual and integrity of the tool use relevant tracts with tool concept scores as a covariate and vice versa. The pattern of results remained overall the same, with effects of all tool concept tracts remaining significant $(p<0.006)$ and the effects of the tool use tracts significant or marginally significant in the lesion analyses $(p<0.10)$ and nonsignificant but in the right direction in the FA analyses ( $p=$ $0.24-0.37$ ). These results suggest that the different mechanisms underlying tool use and concept components extend beyond the motor versus nonmotor distinction.

Dissociation between tool use action and non-tool-use action To investigate whether the effects of the 3 tool use-related tracts were fully explained by their participation in action processing in general, we correlated tool use scores and tract integrity values, with patients' scores on the intransitive action imitation task as a covariate. After controlling for intransitive action imitation scores, tool use scores still significantly correlated with the lesion percentages (partial $r:-0.45$ to $-0.35, p<0.0009$ ) and the mean FA values (partial $r: 0.26-0.30, p<0.02$ ) of all 3 tracts (Table 4 ). 


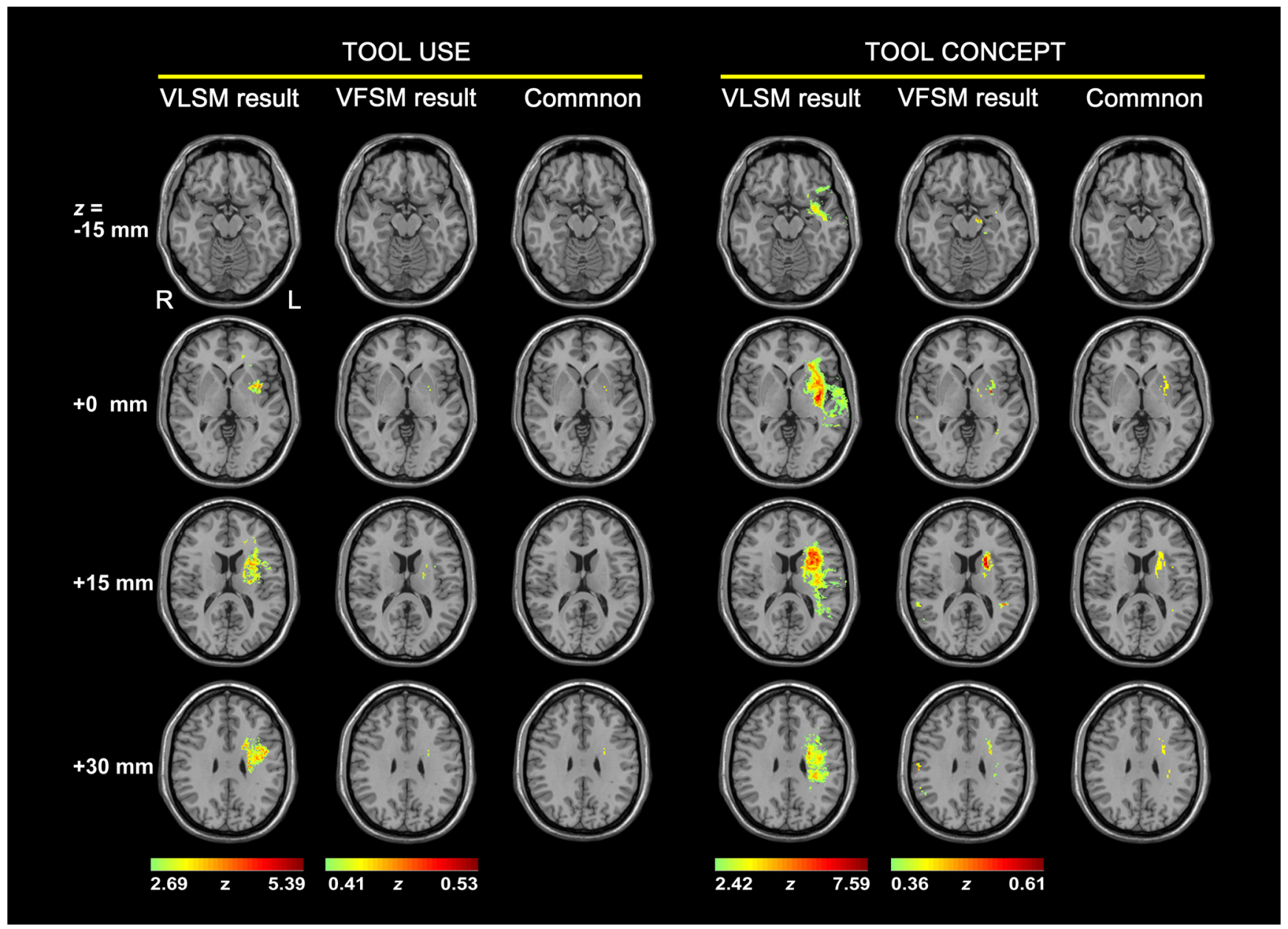

Figure 4. Results of the lesion-symptom mapping analysis for tool processing. For tool use or tool concept processing, Column 1 displays the VLSM (Bates et al., 2003) results, in which tool performance was compared between the damaged and intact groups on each voxel, with total lesion volume regressed out. Column 2 displays the VFSM results, in which tool performance was correlated with FA values on each voxel, with total lesion volume as a covariate. Column 3 shows the voxels with significant effects in both VLSM and VFSM analyses.

Table 4. Partial correlation coefficient for the functional specificity of tool-processing-related tracts, factoring out scores on the controlled variables

\begin{tabular}{|c|c|c|c|c|c|c|c|c|}
\hline \multirow{3}{*}{$\frac{\text { Variable of interest }}{\text { Controlled variable }}$} & \multicolumn{4}{|l|}{ Tool use } & \multicolumn{4}{|l|}{ Tool concept } \\
\hline & \multicolumn{2}{|l|}{ Tool concept } & \multicolumn{2}{|c|}{ Intransitive action } & \multicolumn{2}{|l|}{ Tool use } & \multicolumn{2}{|c|}{ Animal and person concept } \\
\hline & Lesion analysis & FA analysis & Lesion analysis & FA analysis & Lesion analysis & FA analysis & Lesion analysis & FA analysis \\
\hline IDLPMC-IIFG & $-0.38^{* * *}$ & $0.32^{* *}$ & $-0.45^{* * *}$ & $0.26^{*}$ & $-0.28^{*}$ & $0.23^{*}$ & -0.12 & 0.13 \\
\hline IDLPMC-IVPMC & $-0.39^{* * *}$ & $0.34^{* *}$ & $-0.44^{* * *}$ & $0.27^{* *}$ & $-0.24^{*}$ & $0.20 \#$ & -0.12 & 0.13 \\
\hline |IFG-IIPL & $-0.28^{* *}$ & $0.21 \#$ & $-0.35^{* *}$ & $0.30^{* *}$ & $-0.35^{* * *}$ & $0.35^{* * *}$ & $-0.21 \#$ & $0.19 \#$ \\
\hline IIFG-IpMTG & & & & & $-0.47^{* * *}$ & $0.40^{* * *}$ & -0.17 & $0.18 \#$ \\
\hline IIFG-ISPL & & & & & $-0.42^{* * *}$ & $0.35 * * *$ & -0.17 & $0.20 \#$ \\
\hline IIPL-IVPMC & & & & & $-0.28^{* *}$ & $0.29 * *$ & -0.14 & $0.18 \#$ \\
\hline IpMTG-IVPMC & & & & & $-0.33^{* *}$ & $0.33^{* *}$ & -0.16 & $0.20 \#$ \\
\hline ISPL-IVPMC & & & & & $-0.41^{* * *}$ & $0.34^{* *}$ & $-0.21 \#$ & 0.18 \\
\hline
\end{tabular}

Full names of the tract seeds are given in Table 1. $\# p<0.10 ;{ }^{*} p<0.05 ;{ }^{* *} p<0.01 ;{ }^{* * *} p<0.001$

Dissociation between tool conceptual processing and other object categories

We also investigated whether the 8 tracts we found to be crucial for tool concept processing support conceptual processing in general or have (relatively) unique contributions to tools. We performed partial correlations between the integrity scores of these tracts and tool concept composite scores while including conceptual composite scores for the two other semantic categories (animals and persons) as covariates. After controlling for conceptual performance for animals and persons, tool concept composite scores were marginally significantly correlated with the lesion percentage of the IIFG-lIPL tract and the ISPL-IVPMC tracts (partial $r=-0.21, p<0.06$; and for the other 5 tracts: partial $r:-0.17$ to $-0.12, p>0.28)$. In the FA analysis, the effect of the lIFG-lIPL tract (partial $r=0.19, p<0.09$ ) in tool concept processing remained marginally significant, as did the IIFGlpMTG tract (partial $r=0.18, p<0.10$ ), the IIFG-1SPL tract (partial $r=0.20, p<0.07$ ), the IIPL-IVPMC tract (partial $r=$ $0.18, p<0.10$ ) and the lpMTG-IVPMC tract (partial $r=0.20$, $p<0.07$ ) except for the other three tracts (IDLPMC-IIFG: partial $r=0.13, p=0.24$; IDLPMC-IVPMC: partial $r=0.13, p=0.25$; 
ISPL-IVPMC: partial $r=0.18, p=0.10$ ) (Table 4). That is, the IIFG-IIPL tract shows unique contributions for tool concept processing relative to at least two other types of concepts.

\section{Discussion}

We performed deterministic fiber tracking in healthy participants among 14 cortical regions that have consistently been shown to be activated by tool processing and obtained $33 \mathrm{WM}$ tracts connecting these seeds. Three WM tracts connecting left frontoparietal and intrafrontal regions, overlapping with left SLF, are functionally necessary for tool use. These tracts and five additional left hemisphere tracts connecting frontal and temporal/ parietal regions, overlapping with left IFOF, UF, ATR, and SLF, are necessary for normal tool concept processing. The effects of the three tracts in tool use and those of eight tool concept tracts could not be readily accounted for by each other. Whole-brain based VLSM (and VFSM) analyses showed that the brain areas in which the lesion and lower FA values were associated with tool deficits overlapped with major WM pathways in the JHU template: left SLF for tool use; left IFOF, UF, ATR, and SLF for tool concepts. Our findings mapped out the WM structural networks that are functionally necessary for tool use and tool concept processing, respectively.

Although recent studies have also observed structural (Ramayya et al., 2010; Hoeren et al., 2013) and functional (Yoo et al., 2013) connectivity between tool-relevant parietal and frontal lobe regions, we here show directly which of these tracts are in fact necessary for different aspects of tool processing by assessing the effects of damage to these tracts on tool processing performance. Our analyses produced two major results: the anatomical network for tools and the relative dissociation between the tool use and tool concept processing networks, as discussed below.

\section{Tool use structural network}

We observed that the WM connections between left IPL and left IFG and between left DLPMC and left IFG/VPMC (Fig. 3, Table 3 ) are indispensable for patients' tool use ability. The extent of damage to these tracts correlated with the degree of impairment in using tools. The tracts that we identified overlap with the anterior/middle parts of left SLF, which has been shown to be involved in tool use (Ramayya et al., 2010; Hoeren et al., 2013) and various language functions such as syntactic processing (Wilson et al., 2011), phonological encoding in speech production (Schwartz et al., 2012; Han et al., 2014), and verbal working memory (Meyer et al., 2014). The present study further confirms that this tract causally contributes to tool use and, more critically, identifies the exact GM regions being connected by this pathway that contribute to tool use.

These dorsal regions are commonly implicated in various aspects of action processing: IPL is known to be involved in the planning of motor acts (Johnson-Frey et al., 2005), gesture production (Goldenberg and Spatt, 2009; Buxbaum et al., 2014), and the use of objects or judgments of their manipulability (Kellenbach et al., 2003; Rumiati et al., 2004; Boronat et al., 2005; Canessa et al., 2008). Lesions to IPL have been shown to lead to ideomotor apraxia, in which patients can name and understand the function of a tool but do not know how to manipulate it (Heilman et al., 1982; Buxbaum and Saffran, 2002; Goldenberg and Spatt, 2009). The IFG and adjacent middle frontal gyrus regions are also implicated in action production and action understanding (Johnson-Frey, 2004; Lewis, 2006; Goldenberg and Spatt, 2009; Buxbaum et al., 2014) and have been proposed to be relevant for planning, controlling, or representing complex series of motor elements (Liberman and Mattingly, 1985). The premotor cortex is indispensable for producing motor acts, with the dorsal part assumed to be involved in converting knowledge about a motor act into a motor command sequence (Fink et al., 1999; Rumiati et al., 2004) and the ventral part in planning specific arm and hand movements for execution, especially with objects.

The error analyses further indicated that damage to the connection between IFG and IPL tended to be strongly associated with kinematic errors and damage to intrafrontal connections with postural errors. These analyses reveal the different contributions of these tracts in various tool action components. Future studies with more fine-grained tasks directly assessing these specific tool use components are warranted.

\section{Tool concept structural network}

Eight left lateralized WM tracts between the temporal, frontal, and parietal regions were found to be important for tool comprehension (Fig. 3, Table 3). These tasks require knowledge of tool identity, function, or verbal names. This tool comprehension network subsumes the tracts in the tool use network and, in addition, includes five other left hemisphere tracts: lpMTG-IIFG, lpMTG-IVPMC, ISPL-IIFG, ISPL-IVPMC, and IIPL-IVPMC. These additional tracts overlaid with major WM pathways left anterior/middle IFOF, anterior UF, anterior ATR, and SLF, with the former three having been implicated in conceptual processing, independent of input and output modality (Duffau et al., 2005; Schwindt et al., 2013; de Zubicaray et al., 2011; Han et al., 2013a). The tract between pMTG and IPL also showed a strong trend of being important for tool comprehension. The three tooluse-related tracts might participate in tool comprehension by supporting tool use- or action-related knowledge. Their role has been discussed above in the context of tool use and will not be reiterated here.

One hypothesis of the tool concept representation might be that the connections among these regions bind together the action knowledge (frontal and parietal nodes) and the other aspects of tool knowledge, including shape knowledge in the fusiform gyrus and more abstract aspects of function knowledge in pMTG and IPL. It is worth noting that all of the tracts in the tool network were also important for understanding animals and persons, for which the action system may be less central. Of significance is that the tract between IPL and IFG showed greater importance in tool comprehension than in animal and person comprehension, highlighting the central role of this tract in the action system and in tool selectivity. Intriguingly, from the perspective of dualroute models of language, the SLF has been strongly associated with phonological and syntactic processing (see above) and not semantics. The semantic tasks used in previous studies usually included a mixture of stimuli of various domains, which may have obscured the role of the SLF in tool semantics. Therefore, dual-stream models of language (Hickok and Poeppel, 2004) require revision to accommodate the fact that the action aspects of tool semantics are likely to be carried by the "dorsal" language pathway.

\section{Relationship between tool use and tool concept networks}

We here observed partly overlapping networks for tool use and tool concepts: all three tool use tracts were also crucial for tool comprehension, with the latter function involving additional tracts. Partial correlation analyses revealed that these tracts contribute to tool comprehension beyond the extent to which they are important for tool use. Partial correlations in the reverse di- 
rection further suggest that their contribution to tool use cannot be fully explained by the extent to which they represent tool conceptual knowledge. That is, tool use and tool conceptual processing share some, but not all, underlying brain substrates and cognitive mechanisms. These tracts may transfer both manipulation-specific information and more abstract action information about tools, information suitable for representing the causal relationship between tool objects and action goals and functions. In other words, the pattern of results reported here indicates that, whereas tool use information is an integral part of tool concepts, the two types of information are not reducible to each other.

An important caveat to consider is that in the patient analyses the statistical power for each tract varied due to the unequal numbers of patients having a lesion in a given tract. Therefore, when interpreting the effects of tract integrity on tool processing, especially null results, it is important to consider these effects in the light of lesion distributions. Our tool conceptual tasks had more experimental items than the tool use task and thus may have greater sensitivity to reveal subtle impairment severity differences. Given that our tool use task involved actual tool use, it is unclear whether the tool use-relevant connections we found are also important for other tool-related processing such as pantomiming and imitation of tool use. Finally, DTI is an indirect measure of real fiber connections and is especially prone to errors in resolving fiber crossings (Mori and van Zijl, 2002); therefore, convergent evidence from other imaging techniques is warranted. It is worth noting that, although FA values from diffusion imaging may be misleading in voxels with crossing fibers, in the tract integrity-behavior association analyses, the lesion volume measure from structural imaging is immune to this problem. Therefore, results using the combination of these two measures should be taken with greater confidence.

To conclude, by tracking the WM structural pathways between tool-relevant nodes and assessing directly the role of these pathways in tool processing performance, we were able to identify specific frontal-parietal WM tracts that are functionally necessary for tool use and tracts between the frontal, parietal, and temporal regions that are functionally necessary for tool comprehension. The lesion volume and FA values of these tracts significantly predict the severity of tool use and conceptual impairment. These results underscore the causal role of these tracts in tool processing, constituting direct evidence for the anatomical basis of tool processing and specific apraxic or agnosic symptoms.

\section{References}

Bates E, Wilson SM, Saygin AP, Dick F, Sereno MI, Knight RT, Dronkers NF (2003) Voxel-based lesion-symptom mapping. Nat Neurosci 6:448450. Medline

Beauchamp MS, Lee KE, Haxby JV, Martin A (2002) Parallel visual motion processing streams for manipulable objects and human movements. Neuron 34:149-159. CrossRef Medline

Bellec P, Lavoie-Courchesne S, Dickinson P, Lerch JP, Zijdenbos AP, Evans AC (2012). The pipeline system for Octave and Matlab (PSOM): a lightweight scripting framework and execution engine for scientific workflows. Front Neuroinform 6:7. CrossRef Medline

Boronat CB, Buxbaum LJ, Coslett HB, Tang K, Saffran EM, Kimberg DY, Detre JA (2005) Distinctions between manipulation and function knowledge of objects: evidence from functional magnetic resonance imaging. Brain Res Cogn Brain Res 23:361-373. CrossRef Medline

Brunner E, Munzel U (2000) The nonparametric Behrens-Fisher problem: asymptotic theory and small-sample approximation. Biom J 42:17-25. CrossRef

Buxbaum LJ, Saffran EM (2002) Knowledge of object manipulation and object function: dissociations in apraxic and nonapraxic subjects. Brain Lang 82:179-199. CrossRef Medline

Buxbaum LJ, Kyle KM, Menon R (2005) On beyond mirror neurons: inter- nal representations subserving imitation and recognition of skilled object-related actions in humans. Brain Res Cogn Brain Res 25:226-239. CrossRef Medline

Buxbaum LJ, Shapiro AD, Coslett HB (2014) Critical brain regions for toolrelated and imitative actions: a componential analysis. Brain 137:19711985. CrossRef Medline

Canessa N, Borgo F, Cappa SF, Perani D, Falini A, Buccino G, Tettamanti M, Shallice T (2008) The different neural correlates of action and functional knowledge in semantic memory: an FMRI study. Cereb Cortex 18:740-751. CrossRef Medline

Cheng H, Wang Y, Sheng J, Sporns O, Kronenberger WG, Mathews VP, Hummer TA, Saykin AJ (2012) Optimization of seed density in DTI tractography for structural networks. J Neurosci Methods 203:264-272. CrossRef Medline

Crawford JR, Garthwaite PH (2006) Comparing patients' predicted test scores from a regression equation with their obtained scores: a significance test and point estimate of abnormality with accompanying confidence limits. Neuropsychology 20:259-271. CrossRef Medline

Cui Z, Zhong S, Xu P, He Y, Gong G (2013) PANDA: a pipeline toolbox for analyzing brain diffusion images. Front Hum Neurosci 7:42. CrossRef Medline

De Renzi E, Pieczuro A, Vignolo L (1968) Ideational apraxia: a quantitative study. Neuropsychologia 6:41-52. CrossRef

de Zubicaray GI, Rose SE, McMahon KL (2011) The structure and connectivity of semantic memory in the healthy older adult brain. Neuroimage 54:1488-1494. CrossRef Medline

Duffau H, Gatignol P, Mandonnet E, Peruzzi P, Tzourio-Mazoyer N, Capelle L (2005) New insights into the anatomo-functional connectivity of the semantic system: a study using cortico-subcortical electrostimulations. Brain 128:797-810. CrossRef Medline

Fairhall SL, Caramazza A (2013) Brain regions that represent amodal conceptual knowledge. J Neurosci 33:10552-10558. CrossRef Medline

Fink GR, Marshall JC, Halligan PW, Frith CD, Driver J, Frackowiak RS, Dolan RJ (1999) The neural consequences of conflict between intention and the senses. Brain 122:497-512. CrossRef Medline

Folstein MF, Folstein SE, McHugh PR (1975) "Mini-mental state": a practical method for grading the cognitive state of patients for the clinician. J Psychiatr Res 12:189-198. CrossRef Medline

Gao SR (1993) Aphasia. Beijing: Beijing Medicine University and China Xiehe Medicine University Joint Press.

Gibson KR, Gibson KR, Ingold T (1994) Tools, language and cognition in human evolution: Cambridge University.

Goldenberg G (2014) Challenging traditions in apraxia. Brain 137:1858 1859. CrossRef Medline

Goldenberg G, Hagmann S (1998) Tool use and mechanical problem solving in apraxia. Neuropsychologia 36:581-589. CrossRef Medline

Goldenberg G, Spatt J (2009) The neural basis of tool use. Brain 132:16451655. CrossRef Medline

Han Z, Ma Y, Gong G, He Y, Caramazza A, Bi Y (2013a) White matter structural connectivity underlying semantic processing: evidence from brain damaged patients. Brain 136:2952-2965. CrossRef Medline

Han Z, Bi Y, Chen J, Chen Q, He Y, Caramazza A (2013b) Distinct regions of right temporal cortex are associated with biological and human-agent motion: functional magnetic resonance imaging and neuropsychological evidence. J Neurosci 33:15442-15453. CrossRef Medline

Han Z, Ma Y, Gong G, Huang R, Song L, Bi Y (2014) White matter pathway supporting phonological encoding in speech production: a multi-modal imaging study of brain damage patients. Brain Struct Funct. In press. CrossRef Medline

Heilman KM, Rothi LJ, Valenstein E (1982) Two forms of ideomotor apraxia. Neurology 32:342-346. CrossRef Medline

Hickok G, Poeppel D (2004) Dorsal and ventral streams: a framework for understanding aspects of the functional anatomy of language. Cognition 92:67-99. CrossRef Medline

Hoeren M, Kaller CP, Glauche V, Vry MS, Rijntjes M, Hamzei F, Weiller C (2013) Action semantics and movement characteristics engage distinct processing streams during the observation of tool use. Exp Brain Res 229:243-260. CrossRef Medline

Hoeren M, Kümmerer D, Bormann T, Beume L, Ludwig VM, Vry MS, Mader I, Rijntjes M, Kaller CP, Weiller C (2014) Neural bases of imitation and pantomime in acute stroke patients: distinct streams for praxis. Brain 137:2796-2810. CrossRef Medline 
Howard D, Patterson, K (1992) Pyramids and palm trees test: a test of semantic access from words and pictures. Bury St Edmunds, Suffolk: Thames Valley Test Company.

Hua K, Zhang J, Wakana S, Jiang H, Li X, Reich DS, Calabresi PA, Pekar JJ, van Zijl PC, Mori S (2008) Tract probability maps in stereotaxic spaces: analyses of white matter anatomy and tract-specific quantification. Neuroimage 39:336-347. CrossRef Medline

Jefferies E, Patterson K, Ralph MA (2008) Deficits of knowledge versus executive control in semantic cognition: insights from cued naming. Neuropsychologia 46:649-658. CrossRef Medline

Johnson-Frey SH (2004) The neural bases of complex tool use in humans. Trends Cogn Sci 8:71-78. CrossRef Medline

Johnson-Frey SH, Newman-Norlund R, Grafton ST (2005) A distributed left hemisphere network active during planning of everyday tool use skills. Cereb Cortex 15:681-695. CrossRef Medline

Jolliffe IT (2002) Principal component analysis. Berlin: Springer.

Kalénine S, Buxbaum LJ, Coslett HB (2010) Critical brain regions for action recognition: lesion symptom mapping in left hemisphere stroke. Brain 133:3269-3280. CrossRef Medline

Kellenbach ML, Brett M, Patterson K (2003) Actions speak louder than functions: the importance of manipulability and action in tool representation. J Cogn Neurosci 15:30-46. CrossRef Medline

Lewis JW (2006) Cortical networks related to human use of tools. Neuroscientist 12:211-231. CrossRef Medline

Liberman AM, Mattingly IG (1985) The motor theory of speech perception revised. Cognition 21:1-36. CrossRef Medline

Meyer L, Cunitz K, Obleser J, Friederici AD (2014) Sentence processing and verbal working memory in a white-matter-disconnection patient. Neuropsychologia 61:190-196. CrossRef Medline

Mori S, van Zijl PC (2002) Fiber tracking: principles and strategies-a technical review. NMR Biomed 15:468-480. CrossRef Medline

Mori S, Crain BJ, Chacko VP, van Zijl PC (1999) Three-dimensional tracking of axonal projections in the brain by magnetic resonance imaging. Ann Neurol 45:265-269. CrossRef Medline

Oldfield RC (1971) The assessment and analysis of handedness: the Edinburgh inventory. Neuropsychologia 9:97-113. CrossRef Medline

Orban GA, Cauana F (2014) The neural basis of human tool use. Front Psychol 5:310. CrossRef Medline

Ramayya AG, Glasser MF, Rilling JK (2010) A DTI investigation of neural substrates supporting tool use. Cereb Cortex 20:507-516. CrossRef Medline

Randerath J, Goldenberg G, Spijkers W, Li Y, Hermsdörfer J (2010) Differ- ent left brain regions are essential for grasping a tool compared with its subsequent use. Neuroimage 53:171-180. CrossRef Medline

Rorden C, Karnath HO, Bonilha L (2007) Improving lesion-symptom mapping. J Cogn Neurosci 19:1081-1088. CrossRef Medline

Rumiati RI, Zanini S, Vorano L, Shallice T (2001) A form of ideational apraxia as a delective deficit of contention scheduling. Cogn Neuropsychol 18:617-642. CrossRef Medline

Rumiati RI, Weiss PH, Shallice T, Ottoboni G, Noth J, Zilles K, Fink GR (2004) Neural basis of pantomiming the use of visually presented objects. Neuroimage 21:1224-1231. CrossRef Medline

Schwartz MF, Faseyitan O, Kim J, Coslett HB (2012) The dorsal stream contribution to phonological retrieval in object naming. Brain 135:3799_ 3814. CrossRef Medline

Schwindt GC, Graham NL, Rochon E, Tang-Wai DF, Lobaugh NJ, Chow TW, Black SE (2013) Whole-brain white matter disruption in semantic and nonfluent variants of primary progressive aphasia. Hum Brain Mapp 34: 973-984. CrossRef Medline

Smith SM, Jenkinson M, Woolrich MW, Beckmann CF, Behrens TE, Johansen-Berg H, Bannister PR, De Luca M, Drobnjak I, Flitney DE, Niazy RK, Saunders J, Vickers J, Zhang Y, De Stefano N, Brady JM, Matthews PM (2004) Advances in functional and structural MR image analysis and implementation as FSL. Neuroimage 23:S208-S219. CrossRef Medline

Tzourio-Mazoyer N, Landeau B, Papathanassiou D, Crivello F, Etard O, Delcroix N, Mazoyer B, Joliot M (2002) Automated anatomical labeling of activations in SPM using a macroscopic anatomical parcellation of the MNI MRI single-subject brain. Neuroimage 15:273-289. CrossRef Medline

Wang R, Benner T, Sorensen AG, Wedeen VJ (2007) Diffusion toolkit: a software package for diffusion imaging data processing and tractography. Proc Intl Soc Mag Reson Med 15:3720.

Wei T, Liang X, He Y, Zang Y, Han Z, Caramazza A, Bi Y (2012) Predicting conceptual processing capacity from spontaneous neuronal activity of the left middle temporal gyrus. J Neurosci 32:481-489. CrossRef Medline

Wilson SM, Galantucci S, Tartaglia MC, Rising K, Patterson DK, Henry ML, Ogar JM, DeLeon J, Miller BL, Gorno-Tempini ML (2011) Syntactic processing depends on dorsal language tracts. Neuron 72:397-403. CrossRef Medline

Xia M, Wang J, He Y (2013) BrainNet Viewer: a network visualization tool for human brain connectomics. PLoS One 8:e68910. CrossRef Medline

Yoo K, Sohn WS, Jeong Y (2013) Tool use practice induces changes in intrinsic functional connectivity of parietal areas. Front Hum Neurosci 7:49. Medline 